\title{
Dissecting errors of the exogenously-driven and endogenously-driven saccadic tasks in the common marmosets
}

\author{
1 Wajd Amly ${ }^{1}$, Chih-Yang Chen ${ }^{1,2}$, Hirotaka Onoe ${ }^{3}$, Tadashi Isa ${ }^{1,2,3^{*}}$
}

$2 \quad{ }^{1}$ Division of Neurobiology and Physiology, Department of Neuroscience, Graduate School of

3 Medicine, Kyoto University, Kyoto, Japan

$4 \quad{ }^{2}$ Institute for the Advanced Study of Human Biology (WPI-ASHBi), Kyoto University, Kyoto, Japan

$5 \quad{ }^{3}$ Human Brain Research Center, Graduate School of Medicine, Kyoto University, Kyoto, Japan

6 * Correspondence:

7 Tadashi Isa

$8 \quad$ isa.tadashi.7u@kyoto-u.ac.jp

9 Keywords: Callithrix jacchus ${ }_{1}$, gap task $\mathbf{k}_{2}$, oculomotor delayed response task ${ }_{3}$, error trials 4 , trial 10 history ${ }_{5}$.

\section{Abstract}

12 Various saccadic tasks used traditionally in oculomotor research, including both exogenously-driven

13 and endogenously-driven saccades, have been proposed as clinical diagnostic for human movement

14 disorders. Recently, common marmosets have been proven to be a good primate model for these movement disorders. However, whether similar saccadic measurements can be used for marmosets was not tested. Here, we trained three marmosets on the gap task, an exogenously-driven saccadic task, and the oculomotor delayed response (ODR) task, an endogenously-driven saccadic task. We demonstrated that with long-term training, they were able to learn and switch between the two tasks. The marmosets showed undershooting tendency in the gap and both under- and overshooting tendency in the ODR task when they made saccades to the target. We also categorized the error trails into distractive, impulsive, and visuomotor errors, depending on when a false saccade happened concerning the task progression, and interpreted possible causes of errors based on the saccade response time and the task history. Each error category may indicate specific failures in a particular aspect of cognitive or sensorimotor processes. Finally, and critically, we found that saccades from successful trials exhibited the highest saccade peak velocity and the shortest saccade duration comparing to the same saccade amplitude from error trials. Taken together, we showed the potential of training the same marmoset on conflicting oculomotor tasks simultaneously. We documented the baseline performance and compared successful and error trials in both gap and ODR tasks from the same animals. We propose that analyzing the error trials in sync with successful trials could provide a further understanding of the marmosets' cognitive and sensorimotor processes in both healthy and disease conditions. 


\section{$33 \quad 1 \quad$ Introduction}

34 Saccades are conjugated rapid eye movements that primates use to bring their fovea to the target of interest (Yarbus, 1967; Zee and Leigh, 1983). These movements exhibit stereotypic kinematics with a fixed relationship between saccade amplitude, velocity, and duration (Bahill et al., 1975; Lebedev et al., 1996). Besides studying the characteristics of saccade kinematics per se, various saccade paradigms have been developed to probe different neuronal mechanisms (Gooding and Basso, 2008). Several studies have also demonstrated that the saccade kinematics and the measurements of saccade paradigms can be useful in clinical diagnostics of neurological diseases (Leigh and Kennard, 2004; Ramat et al., 2007), including movement disorders (Termsarasab et al., 2015; Waldthaler et al., 2021; Fukushima et al., 2017), psychiatric diseases (Bittencourt et al., 2013; Morita et al., 2020), and neurodegenerative diseases (Antoniades and Kennard, 2015; Opwonya et al., 2021).

To faithfully reproduce these neurological diseases with human-like behavioral symptoms, there are pressing needs to develop animal models using non-human primates (Verdier et al., 2015; Qiu and $\mathrm{Li}$, 2017). The common marmosets (Callithrix jacchus), a new world monkey, has been proposed as a possible primate model for these diseases because of their availability in transgenic tools (Sasaki et al., 2009; Izpisua Belmonte et al., 2015; Park et al., 2016), their smooth brain surface for pinpoint intracranial injection (Lin et al., 2019; Mundinano et al., 2016) and their ability to be trained on behavioral paradigms (Okano, 2021). The marmosets can learn to perform a variety of visual and cognitive tasks under head unrestrained conditions (Spinelli et al., 2004; Clarke et al., 2011; Yamazaki et al., 2011; Jendritza et al., 2021). Their short lifespan also makes them suitable candidates for long-term behavioral observation with the progression of neurological diseases (Tardif et al., 2011). In fact, several neurological models of marmosets have been successfully generated using various methods (Eslamboli et al., 2007; Tomioka et al., 2017; Lin et al., 2019; Jenner et al., 1984; Fox et al., 2010; Emborg, 2017).

The marmosets can also be trained to perform various saccadic paradigms under head restrained conditions (Mitchell et al., 2014), for example, step task (Sedaghat-Nejad et al., 2019; Chen et al., 2021) and gap task (Johnston et al., 2018; Chen et al., 2021). Both tasks are exogenously-driven saccadic tasks because the saccade was initiated in response to a visible visual stimulus. With some modification of the task design, one report has shown successful training for marmosets saccading to a less salient saccade target (anti-saccade task, (Johnston et al., 2019)). However, the endogenouslydriven saccadic tasks, such as oculomotor delayed response (ODR) task or memory-guided saccade task, where the target is only briefly presented and the saccade is initiated to the target location that is no longer visually available (Hikosaka and Wurtz, 1983; Funahashi et al., 1989), were not reported successfully trained on marmosets (but see Carney et al., 2019). Since both exogenously-driven and endogenously-driven saccadic paradigms are important in the clinical diagnostic and for probing different aspects of neurological diseases in humans, the primate model selected for these diseases would be more ideal if the same animal can perform both types of tasks concurrently.

In the current study, we trained three marmosets in two different saccadic paradigms, the gap, and the ODR tasks. We aimed to see if it is possible to achieve a stable performance on the standardized exogenously-driven and endogenously-driven saccade tasks in the same animals. By comparing the task performance, and the saccade kinematics of the two tasks, and identifying the characteristics of the error trials, we have established a baseline measurement to pave the way for future studies of neurological disorders using marmosets as animal models. 


\subsection{Animal ethics}

All marmosets (Callithrix jacchus) used in this study were born in the breeding colony of Kyoto University Animal Research Facility. The experiments followed the guideline of the Japan Neuroscience Society and the Science Council of Japan. Animal Ethics Committee at Kyoto University, Japan approved all experimental procedures.

\subsection{Animal preparation and surgical procedure}

Three adult marmosets were used in this study; marmoset S: male 2.5 years, $390 \mathrm{~g}$; marmoset N: female 2.5 years, $370 \mathrm{~g}$; and marmoset M: male 2 years, $340 \mathrm{~g}$. All marmosets started with one week of transportation cage adaptation followed by two weeks of chair training. After achieving a stable performance in the chair, marmosets underwent surgery of headpost implantation for preparation of head-fixing training. Induction of anesthesia was achieved using $14 \mathrm{mg} / \mathrm{kg} \mathrm{ketamine} \mathrm{and} 0.14 \mathrm{mg} / \mathrm{kg}$ medetomidine along with $0.07 \mathrm{mg} / \mathrm{kg}$ atropine, $0.5 \mathrm{mg} / \mathrm{kg}$ dexamethasone, and $1.5 \mathrm{mg} / \mathrm{kg}$ gentamicin to prevent excessive salivation, relieve pain, and prevent infection. Under $1.5 \%$ isoflurane, each marmoset was aseptically mounted with its own custom-made headpost that we designed based on MRI reconstruction of their skulls (Chen et al., 2021). The headposts were 3D-printed using Dental SG Resin (Form2, Formlabs, U.S.A.), and they were attached to marmosets' skulls using dental resin, Super-Bound (Sun Medical Co., Ltd., Japan). After the surgery, marmosets received 4 days of antibiotics; ampicillin, $30 \mathrm{mg} / \mathrm{kg}$, and anti-inflammatory and analgesics medication; ketoprofen, 0.8 $\mathrm{mg} / \mathrm{kg}$. After medical treatment, the marmosets were given an additional week for recovery.

\subsection{Eye tracking and calibration}

Eyelink 1000 Plus (SR Research, Canada) was used to track marmosets' eyes binocularly with a sampling frequency of $500 \mathrm{~Hz}$. We used both pupil and corneal reflection information for accurate tracking. We calibrated the eyes' position with the same method described in (Chen et al., 2021). Briefly, for online calibration, we used marmoset face photos presented randomly on the screen and the marmosets were free to look at the photos for 10 seconds per trial. We then used a graphical user interface to examine the photos that were presented and the corresponding marmosets' gaze. We manually adjusted the $\mathrm{x}$ and $\mathrm{y}$ gain and the $\mathrm{x}$ and $\mathrm{y}$ offset of the eye positions for each marmoset individually. This calibration was done once for each marmoset after the first time sitting in front of the eye tracker. Then, based on the task performance of the marmosets, slight changes in the gain and offset were made manually each day.

\subsection{Monitor Setup}

We used a 1280 X 1024 pixels resolution LCD monitor at the refresh rate of $60 \mathrm{~Hz}$ (Dell P1917S). The visual distance was $40 \mathrm{~cm}$, and the background where the tasks were presented was a grey background with a luminance of $80.81 \mathrm{~cd} / \mathrm{m}^{2}$. The experimental software used to control the stimuli display was PsychoPy 3.6 (Peirce et al., 2019).

\subsection{Saccadic paradigms}

\subsubsection{Gap task}

In the gap task (Figure 1A), a 1-degree-radius marmoset face picture was presented as a fixation stimulus over a grey background at the center of the screen for up to $1000 \mathrm{~ms}$ at the beginning of a trial. The marmosets were trained to initiate the trial by fixating at the fixation stimulus and staying within a 2-degree-radius invisible window centered at the fixation stimulus (Figure 1A, dashed 
118 circle). The trial started if their gaze entered the window. If they successfully stayed within the

119 window for $200 \mathrm{~ms}$, a gap period was initiated, where the fixation stimulus was removed and only the grey background was presented. During this gap period, the marmosets were trained to keep their gaze within the 2-degree invisible window centered at the removed fixation stimulus. The gap period (0 to $400 \mathrm{~ms}$ ) was predetermined randomly for each session. After successfully finishing the gap period, a peripheral stimulus (1-degree marmoset face) was shown as the saccade target. The target location was randomly picked from eight potential locations separated equally by a 45 -degree radial visual angle from the horizontal meridian with predetermined eccentricity $(4,5,6,7,8$, or 9 degrees) before session initiation. The marmosets were required to make a saccade toward the target and stay within a 2-degree invisible window centered at the saccade target location for $200 \mathrm{~ms}$ to attain a drop $(0.05 \mathrm{ml})$ of the reward. The reward was prepared by mixing baby supplement banana pudding (Kewpie Corp., Japan) with banana-flavored Mei-balance (Meiji Holdings Co., Japan). A 1000 ms was given to the marmosets to make a response, otherwise, the trial was terminated. One session was either 24 successful trails or 48 trials (maximum trial number per session). The marmosets were able to perform $3.20 \pm 0.37$ (mean \pm standard deviation) sessions per day.

\subsubsection{Oculomotor delayed response (ODR) task}

134 In the ODR task (Figure 1B), the marmosets were trained to initiate the trial with similar criteria used in the gap task. After trial initiation, a saccade target was flashed for around $67 \mathrm{~ms}$ and the target location was randomly picked from either right or left relative to the fixation stimulus using predetermined eccentricity $(4,5,6,7$, or 8 degrees) before the session started. A delay period followed immediately after the peripheral saccade target ranging from 100 to $400 \mathrm{~ms}$ (predetermined at session start) in which the marmosets were still required to keep fixating at the fixation stimulus. After the delay period, the fixation stimulus was removed, and the marmosets were trained to make a saccade toward the remembered saccade target location and stay within the 2-degree invisible window centered at the invisible saccade target for $183 \mathrm{~ms}$ to be rewarded. The same response time window of the gap task (1000 ms) was also used here. We used a different fixation and target stimulus (a white circle enclosed by a black arena, total 1-degree-radius, indicated in Figure 1B) than in the gap task to make it easier for the marmosets to learn and to associate each stimulus with each task. One session was terminated if the marmosets reached either 16 successful trails or 32 trials (maximum trial number per session). The marmosets were able to perform $4.05 \pm 1.71$ (mean \pm standard deviation) sessions per day. The gap task was performed first during training.

\subsection{Saccade detection}

We used U'n'Eye, a deep neural network developed for eye movement detection and classification, to detect saccades in our study (Bellet et al., 2019). The eye data were first preprocessed and the ground-truth saccade dataset was first labeled and manually checked following the same procedure and criteria as (Chen et al., 2021). After labeling around 200 trials per animal, the data were gathered and fed into U'n'Eye for network training and validation to get optimal performance for the marmosets. After training, the rest of the preprocessed eye data were fed into the trained network for saccade labeling and the results were inspected manually to check for mislabeling.

\section{$\begin{array}{lll}157 & 2.7 & \text { Successful and error trials definition }\end{array}$}

158 We divided the total trials into either successful or error trials. Successful trials were defined as the

159 trials that the marmosets made saccades toward the target and stayed within a 2-degree invisible

160 window centered at the saccade target location for $200 \mathrm{~ms}$ or $183 \mathrm{~ms}$ in the gap or ODR tasks, 161 respectively, and obtained the reward at the end of the trials. Alternatively, error trials were defined 
162 as the trials that the marmosets failed to get the reward at the end of the trials. A detailed definition of

163 the error trials is mentioned in the Result section.

\section{$164 \quad 2.8 \quad$ Data analysis}

\section{$165 \quad$ 2.8.1 Task performance and stability}

166 To compare the improvement of each task before and after long-term training, we calculated the

167 success rate of the first and the last month of training for the gap task with each gap period and the

168 ODR task with each delay period regardless of the target eccentricities for each marmoset. We

169 further analyzed if the success rate was dependent on target eccentricity by separating the successful

170 trials into the first and the latter half according to the training period for each task and each animal

171 regardless of the gap or delay period. We then took the difference between the first the latter half of

172 the success rate and combined the result of the three marmosets to analyze if the performance

173 improvement was correlated with the target eccentricity for each task. The same practices were

174 performed to determine if the success rate changed with the gap or the delay period for each task and

175 whether the improvement was correlated with the gap or delay period.

176 To analyze the performance stability, we also compared the standard deviation (std) of the success

177 rate of the first and the last month of training for the gap task with each gap period and the ODR task

178 with each delay period regardless of the target eccentricities for each marmoset. We took the

179 difference of the std calculated according to the target eccentricity between the first and the latter half

180 of the training period again to check if the change in standard deviation was dependent on the target

181 eccentricity for each task. The same practice was again taken to determine the correlation between

182 the change in std and the gap or delay period.

\section{$183 \quad$ 2.8.2 Radial distance from the saccade endpoint to the target location}

184 To analyze whether the marmosets had any systematic saccade undershooting (hypometria) or 185 overshooting (hypermetria) when they made their saccades towards the targets in each task, we 186 measured the radial distance from the saccade endpoint to the target location. We first rotated all the 187 target locations and the saccades after target onset in the gap task or fixation stimulus offset in the

188 ODR task. The rotation angle was determined by the angle between the target location of the trial and 189 the positive x-axis. Next, we subtracted the length of the saccade after rotation to the distance of the 190 rotated target location to obtain the radial distance.

\section{$191 \quad$ 2.8.3 Saccade main sequence}

192 To characterize the change of saccade kinematics for different trial categories, we looked at the 193 saccade main sequence (Bahill et al., 1975; Chen et al., 2021). We first plotted the saccade amplitude 194 (x) against the saccade peak velocity $(\mathrm{f}(\mathrm{x}))$. We then fitted the data with the following equation:

$$
f(x)=a \times\left(x^{n}\right)
$$

195 a and $\mathrm{n}$ are the fitting parameters representing the slope and the exponent.

196 We also plotted the saccade amplitude (x) against the saccade duration $(\mathrm{f}(\mathrm{x}))$ and fitted the data with 197 the following equation:

$$
f(x)=b \times(x)+c
$$


$\mathrm{b}$ and $\mathrm{c}$ are the fitting parameters representing the slope and the intercept. All fitted curves were overlaid on the correspondent figure, color-coded with trial categories.

\subsection{Statistical analysis}

201 All statistical analysis is described in the Result section. Additional statistical analysis was performed

202 and described in the Supplementary Figures. If not specifically mentioned, the result of the analysis

203 was made by combining all three marmosets' data.

\section{Result}

\section{$205 \quad 3.1 \quad$ Task performance improved after long-term training}

All three marmosets were trained for eight months on both gap and oculomotor delayed response (ODR) tasks (Figure 1). To quantify the effect of training, we separated the trials into successful and error trials, based on if the marmosets were rewarded or not while performing the tasks (Materials and Methods). When the training just started for one month, the successful trials were on average $56.0 \%$ across the marmosets in the gap task and $21.0 \%$ in the ODR task (Figure 2). This indicates that likely the gap task was intrinsically easier than the ODR task for the marmosets. At the last month of training, the successful trials increased on average to $75.7 \%$ in the gap task (marmoset $\mathrm{S}: \mathrm{p}$ $=0.004$; marmoset $\mathrm{N}: \mathrm{p}=0.08$; marmoset $\mathrm{M}: \mathrm{p}=2 \times 10^{-13}$, Wilcoxon rank sum test). However, the successful trials did not increase significantly in the ODR task (average, 24.0\% marmoset $\mathrm{S}: \mathrm{p}=0.6$; marmoset $\mathrm{N}: \mathrm{p}=0.2$; marmoset $\mathrm{M}: \mathrm{p}=0.1$, Wilcoxon rank sum test). There were no clear correlation between the success rate and the target eccentricity either in the first 4 months (gap: marmoset $S$ : rho $=-0.8, \mathrm{p}=0.06$; marmoset $\mathrm{N}$ : rho $=-0.9, \mathrm{p}=0.02$; marmoset $\mathrm{M}$ : rho $=-0.6, \mathrm{p}=0.2$, ODR: marmoset $\mathrm{S}$ : rho $=-0.2, \mathrm{p}=0.9$; marmoset $\mathrm{N}$ : rho $=0.5, \mathrm{p}=1$; marmoset $\mathrm{M}$ : rho $=0.5, \mathrm{p}=1$; Spearman Rank coefficient) or in the latter 4 months of training, in both tasks (gap: marmoset $S$ : rho = $-0.3, p=0.7$; marmoset $\mathrm{N}$ : rho $=0.09, \mathrm{p}=0.9$; marmoset $\mathrm{M}$ : rho $=-0.9, \mathrm{p}=0.03$, ODR: marmoset $\mathrm{S}$ : rho $=-0.6, \mathrm{p}$ $=0.4$; marmoset $\mathrm{N}$ : rho $=1, \mathrm{p}=0.3$; marmoset $\mathrm{M}$ : rho $=1, \mathrm{p}=0.3$; Spearman Rank coefficient). The improvement comparing the first half of the training to the latter half was also not correlated with the target eccentricity (gap: rho $=0.3, \mathrm{p}=0.2$, ODR: rho $=0.3, \mathrm{p}=0.5$; Spearman Rank coefficient). Similarly, the correlation between the success rate and the gap or the delay period was weak in the first half (gap: marmoset $\mathrm{S}$ : rho $=-0.1, \mathrm{p}=0.8$; marmoset $\mathrm{N}$ : rho $=0.5, \mathrm{p}=0.4$; marmoset $\mathrm{M}$ : rho = $0.2, \mathrm{p}=0.4$; Spearman Rank coefficient, ODR: marmoset $\mathrm{S}$ : rho $=-1, \mathrm{p}=0.003$; marmoset $\mathrm{N}$ : rho = 0.7, $\mathrm{p}=0.2$; marmoset M: rho $=0.03, \mathrm{p}=1$; Spearman Rank coefficient) and also in the later half, (gap: marmoset $\mathrm{S}$ : rho $=0, \mathrm{p}=1$; marmoset $\mathrm{N}$ : rho $=-0.6 \mathrm{p}=0.2$; $\operatorname{marmoset} \mathrm{M}$ : rho $=-0.8, \mathrm{p}=$ $8.3 \times 10^{-4}$, ODR: marmoset $\mathrm{S}$ : rho $=-0.9, \mathrm{p}=0.007$; marmoset $\mathrm{N}$ : rho $=-0.4, \mathrm{p}=0.5$; marmoset $\mathrm{M}$ : rho $=-0.8, \mathrm{p}=0.03$; Spearman Rank coefficient) of the training. However, the improvement between the first and the latter half of the training was correlated with the gap period and was weaker in longer gap period in the gap task (rho $=-0.6, p=0.02$; Spearman Rank coefficient). In the ODR task, the improvement was not correlated with the delay period (rho $=-0.13, \mathrm{p}=0.6$; Spearman Rank coefficient). We also found that the averaged standard deviation (std) of the success rate decreased from 22.4 to 17 in the gap task (marmoset $\mathrm{S}: \mathrm{p}=2.3 \times 10^{-19}$, marmoset $\mathrm{N}: \mathrm{p}=0.6$, marmoset $\mathrm{M}: \mathrm{p}=$ $3 \times 10^{-21}$, Two-sample F-test) and 16.3 to 10.6 in the ODR task (marmoset $\mathrm{S}: \mathrm{p}=3 \times 10^{-4}$; marmoset N: $\mathrm{p}=0.6$; marmoset $\mathrm{M}: \mathrm{p}=9.2 \times 10^{-5}$, Two-sample F-test) comparing the first to the last month of the training period (Figure 2). This indicates that the task performance became more stable after longterm training for both tasks. The decrease in std was not correlated with either the target eccentricity (gap, rho $=-0.3, \mathrm{p}=0.3$, ODR, rho $=0.4, \mathrm{p}=0.4$, Spearman Rank coefficient) or the gap/delay period (gap, rho =0.14, $\mathrm{p}=0.6$; ODR, rho $=-0.4, \mathrm{p}=0.1$, Spearman Rank coefficient). Besides longterm training, another possible reason for the improvement in the task performance was that for 
simplicity of training, we used only two possible target locations (horizontal left or right) in the ODR task instead of eight in the gap task (Materials and Methods). The marmosets could have memorized the possible target locations and randomly chose either one. Indeed, we found that in the error trials, there were $6.1 \%$ of the trials where the marmosets picked the exact opposite side of the target location in the last month of training. This was higher than in the first training month (4.5\%). However, this probability was still lower than the success rate in the last month of training $(24.0 \%)$. Based on the above evidence, we believe that the marmosets learned the task structure and the possible locations of the target, and they were using the cue as a guide instead of only guessing where the target would be in the ODR task. Hence, the marmosets started with relatively low and unstable task performance in both the gap and ODR tasks, but after long-term training, they improved their performance in both the success rate and the performance stability, especially in the gap task. They were also able to generalize such improvements in different task conditions, for instance, target eccentricity and gap/delay periods starting from the beginning of the training.

\subsection{Hypometric saccades in both tasks but hypermetric saccades only in ODR task found}

To further investigate how the marmosets reached the target in either task, we plotted the radial distance of their very first saccade after target onset in the gap task or fixation spot offset in the ODR task from the successful trials (Figure 3A). In the gap task, we found that most of these saccades landed within the target window (Figure 3B). However, a small portion of these saccades were hypometric and the portion increased with target eccentricity (rho $=0.9, \mathrm{p}=1.9 \times 10^{-9}$; Spearman Rank coefficient), but not with gap period ( $r h o=0.2, p=0.2$; Spearman Rank coefficient). If a second saccade was made following the first, the endpoint of the second saccade was in general closer to the target (Figure 3B, $\mathrm{p}<2.2 \times 10^{-308}$; Wilcoxon rank-sum test). The occurrence of the second saccades were more frequent with larger eccentric target (rho $=0.8, \mathrm{p}=2.8 \times 10^{-4}$; Spearman Rank coefficient) and with longer gap period (rho $=0.4, \mathrm{p}=0.02$; Spearman Rank coefficient). This means that in some trials, particularly for larger eccentric targets, the marmosets made several short saccades (hypometric saccades) to reach the target in the gap task.

In the ODR task, the hypometric saccades did not increase significantly either with target eccentricity (rho $=0.5 \mathrm{p}=0.1$; Spearman Rank coefficient) or with the delay period ( (ho $=-0.05, \mathrm{p}=0.8$; Spearman Rank coefficient) (Figure 3C). On the other hand, hypermetric saccades were found (Figure 3C), which was not observed in the gap task. The percentage of hypermetric saccades was decreasing with the larger eccentric target ( $r h o=-0.9, p=8.5 \times 10^{-7}$; Spearman Rank coefficient). If a second saccade was made following the first, again, the endpoint of the second saccade was closer to the target than the first saccade $\left(\mathrm{p}=1.6 \times 10^{-22}\right.$; Wilcoxon rank-sum test $)$. The occurrence of second saccades was not significantly correlated with target eccentricity (rho $=-0.5, \mathrm{p}=0.09$; Spearman Rank coefficient), but it was less frequent compared to the correspondent eccentricity in the gap task ( $\mathrm{p}=2.4 \times 10^{-4}$; Wilcoxon signed-rank test).

Having found successful trials with hypermetric and hypometric saccades, we further subdivided them into three subgroups: pure single saccade group, single saccade group, and multiple saccades group, based on how the marmosets reached the target (Figure 4). We next defined the saccade that started within the fixation window and ended within the target window as the "answering saccade" (from pure single saccade and single saccade group, Figure 4A, Figure 3D) and replotted the distance between the answering saccade endpoint to the target (Figure 3E, F). To assess the accuracy of these answering saccades, we calculated the half-width of the histograms in Figure 3E, F as a proxy of it. The half-width did not change with eccentricity in the gap task (rho $=0.5, \mathrm{p}=0.05$; Spearman Rank coefficient) and was, in general, smaller than those in the ODR task $(\mathrm{p}=0.02$; 
Wilcoxon signed-rank test). However, the half-width decreased with eccentricity in the ODR task (rho $=-0.8, \mathrm{p}=2.9 \times 10^{-4}$; Spearman Rank coefficient), suggesting that the accuracy of the answering saccades was higher with larger eccentric targets. For the saccade following the answering saccade, again, we found that the endpoint of this saccade was closer to the target than the answering saccade (gap / ODR; $\mathrm{p}<2.2 \times 10^{-308} / \mathrm{p}=1.2 \times 10^{-5}$; Wilcoxon rank-sum test), and the second saccades were less frequent in the ODR task than in the gap task $\left(\mathrm{P}=2.4 \times 10^{-4}\right.$; Wilcoxon rank-sum test $)$. We also analyzed separately the $\mathrm{x}$ and $\mathrm{y}$ distance of the answering saccades endpoints and confirmed that $\mathrm{x}$ offsets were always larger than y offsets (Supplementary Figure 1).

\section{6}

\subsection{Effects of gap period}

After defining the answering saccades, we calculated the saccade response time (SRT) of saccades as the time difference between the target onset in the gap task or fixation offset in the ODR task and the starting time of the saccades. As indicated in Figure 5A, the SRT decreased with increasing gap period (rho $=-0.4, p=0.03$; Spearman Rank coefficient). This comes in agreement with the gap effect phenomenon, defined as a temporal gap between fixation point offset and target onset that typically results in shorter saccadic latencies than if the fixation point remains on (Saslow, 1967; Reulen, 1984; Tam and Ono, 1994; Pratt et al., 1999; Chen et al., 2021). This effect was not dependent on the eccentricity of the target location ( $r h o=-0.06, p=0$. 8; Spearman Rank coefficient). Furthermore, a detailed analysis of the SRT distributions for all three marmosets showed no express saccades in the gap task (Supplementary Figure 2A).

\subsection{Potential capture of attention effect in ODR task}

As for the ODR task (Figure 5B), a decreasing trend of SRT with increasing delay period was observed only in one marmoset (marmoset M: rho $=-0.9, \mathrm{p}=0.02$; Spearman Rank coefficient). However, the other two marmosets showed a decreasing trend of SRT up until $200 \mathrm{~ms}$ then a rebound of slower SRT at $250 \mathrm{~ms}$. The averaged SRT across the three marmosets did not show a clear trend of decreasing SRT with increasing the delay period ( $r h o=-0.1, p=0.6$; Spearman Rank coefficient). Similar to the gap task, there was also no dependency of the SRT on the eccentricity of the target location (rho $=-0.5, \mathrm{p}=0.1$; Spearman Rank coefficient). In addition, the averaged SRT of gap / ODR task was 155 / 149 ms for Marmoset S, 165 / 240 ms for Marmoset N, and 158 / 194 ms for Marmoset M. This result showed that the SRT in the ODR task was generally longer than that for the gap task for the same marmoset (marmoset $\mathrm{S}: \mathrm{p}=1.5 \times 10^{-21}$, marmoset $\mathrm{N}: \mathrm{p}=1.6 \times 10^{-80}$, marmoset $\mathrm{M}$, $\mathrm{p}=5.6 \times 10^{-37}$; Wilcoxon rank-sum test). We also noticed some ultra-short latency answering saccades exhibited in the ODR task (Supplementary Figure 2B), which was consistent across all three marmosets.

\section{1}

\subsection{Different types of errors made by the marmosets}

In addition to the successful trials, the marmosets also made many errors during the tasks (error rates; gap task: $37.7 \%$, ODR task: $78.7 \%$, averaged from three marmosets). Therefore, we decided to take benefit from these errors and try to interpret why the marmosets ended up not getting the reward. To do so, we divided the error trials into three categories, distractive error, impulsive error, and visuomotor error categories, based on when the marmosets made mistakes relative to the task monitor events (Figure 6A). For the gap task, we first labeled the error trials based on three monitor events in a given trial, fixation stimulus onset, fixation stimulus offset, and target stimulus onset. If a trial ended after fixation stimulus onset and before fixation stimulus offset, it was defined as a distractive error trial. If a trial ended after fixation stimulus offset and before target stimulus onset, it was defined as an impulsive error trial. Finally, if a trial ended after target stimulus onset but without 
a reward, it was defined as a visuomotor error trial. We then looked for the first saccade in the labeled trial which broke fixation (i.e., the first saccade started within the fixation window and ended outside the fixation window). This saccade was taken as the saccade that responded to the nearest monitor event for this trial, and the SRT was then calculated. In the gap task, anticipatory saccades were reported for marmosets (Chen et al., 2021). These anticipatory saccades are considered not responding to the nearest monitor event (target onset) because the response time was too short $(<70$ $\mathrm{ms}$ ). They are likely the slower saccades responding to the second nearest monitor event (fixation offset). Considering that, we redefined visuomotor error trials with SRT less than $70 \mathrm{~ms}$ to be impulsive error trials and redefined impulsive error trials with SRT less than $70 \mathrm{~ms}$ to be distractive error trials and recalculated the SRT based on the second nearest monitor events. If the marmoset never entered the fixation eye window after fixation stimulus onset, the trial was defined as a distractive error trial and no saccade or SRT was identified. Also, if the marmoset did not leave the fixation window after entering it, the trial was defined as a visuomotor error trial and no saccade or SRT was identified. Example trials are shown in Figure 6B.

The definitions of the error categories were similar in the ODR task (Figure 6C). The three monitor events were identified as fixation stimulus onset, target stimulus onset, and fixation stimulus offset. Distractive error trails were then defined as the trials ended after fixation stimulus onset and before target stimulus onset. Impulsive error trials were defined as trials that ended after target stimulus onset and before fixation stimulus offset. Visuomotor error trials were defined as trials ended after fixation stimulus offset but without a reward. Again, the SRT was extracted for each trial. No anticipatory saccades were reported previously, so we did not relabel any trials. Example trials are shown in Figure 6D.

\subsection{Distinct error rate and saccade response time for each category}

We first compared the distractive error category in either task (Figure 7). Because the marmosets were trained with different fixation stimulus configurations (Figure 1A), they should be able to distinguish the tasks at the very beginning of the tasks. This was reflected by different distractive error rate. We expected that because the ODR task was more demanding and had a lower success rate, the distractive error rate in the ODR task should be higher. However, to our surprise, the result was the opposite: the distractive error rate was lower in all three marmosets in the ODR tasks (marmoset $\mathrm{S}: \mathrm{chi}^{2}=34.2, \mathrm{p}=5 \times 10^{-9} ;$ marmoset $\mathrm{N}: \mathrm{chi}^{2}=1.3 \times 10^{3,} \mathrm{p}<2.2 \times 10^{-308}$; marmoset $\mathrm{M}: \mathrm{chi}^{2}=$ 677.5, $\mathrm{p}<2.2 \times 10^{-308}$; chi-square test).

363 For the impulsive error category, we found that the error rate was much higher in the ODR than in

364 the gap task (Figure 7). This was probably because the upcoming target location was not provided during the gap period, whereas it was displayed briefly just before the delay period in the ODR task. The marmosets needed to actively suppress their reflexive response to the target, and failure to suppress such impulsivity would result in breaking fixation faster than in the gap task. Indeed, the SRT in the impulsive error category was shorter in the ODR than in the gap task (marmoset $\mathrm{S}: \mathrm{p}=$ $5 \times 10^{-27}$, marmoset $\mathrm{N}: \mathrm{p}=1 \times 10^{-105}$, marmoset $\mathrm{M}, \mathrm{p}=6.9 \times 10^{-264}$; Wilcoxon rank-sum test) and was comparable with that in the successful trials of the gap task (Figure 8).

We also found that the visuomotor error rate was higher in the ODR than in the gap task (Figure 7), and the SRTs in the visuomotor error category were slower in the ODR than in the gap task (marmoset $\mathrm{S}: \mathrm{p}=9.3 \times 10^{-119}$, marmoset $\mathrm{N}: \mathrm{p}=7.7 \times 10^{-92}$, marmoset $\mathrm{M}, \mathrm{p}=1.5 \times 10^{-153}$; Wilcoxon ranksum test) (Figure 8). The SRTs were also longer in the visuomotor error trials than in the successful 
375 trials (marmoset $\mathrm{S}: \mathrm{p}<2.2 \times 10^{-308}$, marmoset $\mathrm{N}: \mathrm{p}=5.4 \times 10^{-104}$, marmoset $\mathrm{M}, \mathrm{p}<2.2 \times 10^{-308}$;

376 Wilcoxon rank-sum test) (Figure 8).

\section{$377 \quad 3.7 \quad$ Marmosets repeated the same incorrect answer in the ODR task}

378 To further investigate why the marmosets failed in each step of the ODR task, we separated the

379 saccade vectors made relative to the target location in the impulsive and the visuomotor error

380 categories (Figure. 9A). We found that across all three marmosets, saccades from the impulsive error

381 trials were mainly directed towards the target stimulus. This implies that the marmosets were indeed

382 incapable of suppressing their reflexive saccade toward the briefly presented target. From the

383 visuomotor error trials, the main failure reason for saccades directed towards the target was the

384

385

386

387

388

389

390

391

392

393

394

395

396

397

398 generation of hypometric saccades that did not reach the target window (Figure. 9B). However, the marmosets made more often, saccades away from the target stimulus (Figure. 9A). This was an unexpected result because if the marmosets forgot the target direction of the current trial, an equal bias of saccading toward or away from the target would've been seen. If the marmosets only remembered the rough target direction but not the exact location, the saccade direction should be made towards the target location. To tackle this question, we took the saccades directed away from the target, of the visuomotor error trial and named them as "current trial saccades" and inspected what the marmosets' previous responses were (Figure 9C). We found that if the previous trial was successful, the marmosets did not show any tendency to select the same or the opposite location of the target that they chose before (Figure 9C, left figure). However, if the previous trial was impulsive, the marmosets showed a tendency to saccade to the opposite side of what their previous choice was (Figure 9C, middle figure). This means that the marmoset actually made a saccade away from the target in the current visuomotor trial given that most of the impulsive error trails had saccades directed toward the target (Figure 9A). Finally, if the visuomotor error trail was preceded by another visuomotor trial, the marmosets tended to stick with the same answering direction of their choice (Figure 9C, right figure), which would be away from the target location.

3.8 High saccade peak velocity and short saccade duration for successful answering saccades

401 Finally, we compared the saccade kinematics across all categories for both tasks. We first looked at the relationship between saccade peak velocity and amplitude. As Figure 10A, B shows, in both the gap task and the ODR task, the peak velocity was similar in the successful trials and was also the highest comparing to saccades in other error categories. Furthermore, we found that the saccades' duration in the successful trials was the shortest compared to the error saccades in either task when looking at the relationship between saccade duration and amplitude (Figure 10C, D).

\section{Discussion}

We showed that the marmosets were able to learn two different saccadic paradigms, the gap, and the ODR task, and switch in between them. Regarding successful trials, we found hypometric saccades in the gap but both hypo- and hypermetric saccades in the ODR task. We also analyzed the SRT and confirmed the gap effect and potential capture of attention in either task from the marmosets. We further categorized and analyzed the error trials to interpret why the marmosets made mistakes in each task. We found that the marmosets had fewer distractive errors in the ODR task, however, the impulsive errors were more compared to the gap task. Although there were more visuomotor errors in the ODR than in the gap task, this was not an outcome of a previous answer influence. Rather, these errors might be the result of repetitively making the same mistake. In the end, we found that the successful trials had the highest saccade peak velocity and the shortest saccade duration under similar saccade amplitude in both tasks. To the best of our knowledge, no previous research has fully 
419

420

421

422

423

424

425

426

427

428

429

430

431

432

433

434

435

436

437

438

439

440

441

442

443

444

445

446

447

448

449

450

451

452

453

454

455

456

457

458

459

460

461

462

463

464 documented the learning curve of the gap and ODR tasks and directly compared the two in the same marmoset. This is also the first time a detailed analysis was made in both the successful and the error trials to interpret the potential cognitive and sensory-motor processes while the marmosets were performing the tasks. We believe our approach can better evaluate the marmosets' behavior and pave the way to analyze the behavior in both healthy and disease model marmosets.

We started the comparison by looking at the success rate in each task. Our data showed clearly that the marmosets learned both tasks and were able to switch in between them. It is known that longterm training helps in improving the subject's ability to correctly respond to visual stimuli (Goldstone, 1998). But, if we cross-compared between the two tasks, the success rate was lower in the ODR task than in the gap task (Figure 2). We attribute this to the complete reliance on working memory in recalling the location of the previously presented target in the ODR task. Indeed, previous macaque monkey studies have shown that saccades made in response to a remembered target location are associated with larger errors than saccades made in response to a visually accessible target (Opris et al., 2003; Stanford and Sparks, 1994). There might be also interference between the exogenouslydriven and endogenously-driven saccadic tasks being trained in parallel. The marmosets might have chosen to receive more rewards on the easier task but avoid doing the difficult one. However, in the ODR task, there was no clear avoidance behavior (for example, sudden performance drop), and after long-term training, there was a performance improvement, although not as dramatic as in the gap task.

Next, we looked at the accuracy of the first saccade and the answering saccade after target onset. As our data showed, the marmosets have the tendency to undershoot in their first saccade in the gap task, and they make a following saccade that corrects for it (Figure 3). This comes in line with the previous human research that visually guided saccade tasks tend to have answering saccade first undershooting and frequently followed by a corrective secondary saccade to line up the fovea with the target (Nuthmann et al., 2016). This undershooting is referred to as an attempt to reduce the saccade flight time (Harris, 1995). For the ODR task, less corrective saccade occurred because there was no visual target to align with. However, there was a clear saccadic range effect with saccades biased toward the center of the range, undershooting for furthest targets from the fixation center, and overshooting for closest targets to the fixation center. (Kapoula, 1985). This is mainly occurring due to the task condition. Nonetheless, if we looked at the half-width of the answering saccade distribution, we found that the 5 deg target was the narrowest. This may indicate that 5 deg target was the most precisely encoded target in the ODR task. Additionally, although previous research showed that memory-guided saccade is usually more hypometric than visually guided saccades (Nuthmann et al., 2016), a similar effect was not observed in our current study.

We did not find express saccade in gap task in the current study (Figure 4), in contrary to our previous study (Chen et al., 2021). Even though the training for gap task was almost identical to our previous report. We attribute this difference to two possible reasons, one is the interference from the ODR task, and the other is the use of multiple training eccentricities. Furthermore, not all marmosets showed express saccade after gap task training (Johnston et al., 2018). But we did observe the gap effect in the current study. On the other hand, ultra-short latency answering saccades were found in the ODR task, which can be a result of several possible cognitive elements such as predictions, knowledge, and strategies (Badler and Heinen, 2006; Heeman et al., 2019). In addition, when we compared the gap and ODR tasks, we found that in general, the SRT in the ODR task was longer than in the gap task in the same marmoset (Figure 5). This also comes in accordance with previous macaque studies which have shown that the response time of memory-guided saccades is longer and less accurate than visually guided saccades (Gnadt et al., 1991). We also found that the SRT from 
two marmosets in the ODR task decreased until $200 \mathrm{~ms}$ and then increased. This is analogous to the capture of attention effect observed in the Posner paradigm (Posner, 1980). However, because the ODR task and the Posner paradigm are different, and not all marmoset showed the same response, it is hard to make a solid conclusion.

469 One important point of the current study was to extend the analysis to include the error trials and to interpret how the marmosets made mistakes (Figure 6). First, we found that the distractive error rate was lower in the ODR task in contrast to the gap task (Figure 7). This is similar to "mindwandering", where attention is generally directed to task-irrelevant internal thoughts for easy and repetitive tasks (Reichle et al., 2010). Moreover, many other studies proposed that whenever internal cognition is activated, eyes are disengaged from the external stimulus in order to guard the internal thoughts against external events (Smallwood et al., 2007). To successfully execute a demanding task, it is a must to stay concentrated on the current ongoing task and not going to the mind-wandering state (Benedek et al., 2017). Therefore, the lower distractive error in the ODR task might be due to the higher demands of the task, which activates more cognitive processes. However, the gap task is mainly driven by external stimulus and could lead to a mind-wandering state easily. We also found that the impulsive error rate was higher in the ODR task. During the gap period, there was no information about the target, hence, the impulsive error rate was lower in the gap task. Even if there was an impulsive error, because the marmosets did not know where to saccade to, the SRT was much slower than the ODR task. Yet, the SRT of the impulsive errors in the ODR task was similar to the SRT of the answering saccades in the gap task. This might be because the marmosets reflexively made saccades towards the flashed target, and they ignored the fixation spot almost completely (Figure 8). This could be a consequence of our parallel training of both tasks. Furthermore, the visuomotor error rate was higher in the ODR task. This could be related to the task design. Because in the gap task, the final stage was to make an exogenous saccade to a target stimulus, whereas in the ODR task, marmosets must rely on their working memory to recall the location of the target and generate an endogenous saccade towards an invisible stimulus. Because of this, the SRT was also slower in the ODR task for visuomotor errors. At last, we noticed that a good proportion of the successful answering saccades and the saccades in visuomotor errors in the ODR task had very short response times, but not in the gap task. This might be due to the strategy that the marmosets used to answer in the ODR task. In particular, the same delay period was used in the same session, which may have reinforced the marmosets to count the time.

We noticed that in the ODR task, visuomotor error trials' SRTs were consistently longer than successful trials' SRTs. This might indicate that the marmosets did not remember the target location and hesitated in making a saccade to the invisible target. Therefore, we further dissected how the marmosets made a visuomotor error in the ODR task by considering their previous decision (Figure 9). Since the target was either presented left or right and to prevent the marmosets from picking only one side to get the reward, the failed trial in our task repeated itself until the marmosets successfully answered it, we wondered if their decision in the previous trial might have influenced their current responses. However, we did not find evidence that the previous successful trial affected the marmoset's upcoming decision. However, if the marmosets made a visuomotor error previously, they tended to repeat the same answer. This may be similar to an effect called "recurring mistakes" (Kuvaldina et al., 2019) where the same mistakes are repetitively made after the first mistake. Although the neuronal pathway of recurring mistakes is still not clear, our experiment suggests that the marmosets might be useful to identify the neuronal bases of this psychological phenomenon using the ODR task. 
510

511

512

513

514

515

516

517

518

519

520

521

522

523

524

525

526

527

528

529

530

531

532

533

534

535

536

537

538

539

540

541

542

543

544

545

546

547

548

549

The main sequence relationships are believed to reflect optimization of the trade-off between saccade duration and accuracy (Harris and Wolpert, 2006). It is commonly known that there is a fixed relationship between the saccade amplitude to the peak velocity and duration (Fuchs, 1967; Bahill et al., 1975). But when comparing visually guided and memory-guided saccades in macaque, it has been shown that saccades made in response to remembered locations have lower peak velocities and longer durations than saccades made in response to a visible stimulus (Gnadt and Andersen, 1988; Powers et al., 2013). In our results (Figure 10), we found that the previous statement is true only for saccades with small amplitude, however, as saccade amplitude got larger, answering saccades made in the ODR task had higher peak velocities and shorter durations than the amplitude of the same saccade made in the gap task. Our data also showed that the amplitude vs duration relationship had a slope smaller in the ODR task than in the gap task. This could be due to motivation, arousal, or advanced motor planning. It has been recently noticed that saccade peak velocity is not just dependent on saccade amplitude, but also on motivation, arousal, and inter and intra-individual variations (Muhammed et al., 2020). In addition, for advanced motor preparation, the expected value comes with great significance and, various studies have shown that saccade velocity can actually increase if a high reward was anticipated (Di Stasi et al., 2011; Chen et al., 2013). We think that motivation and advanced motor planning play crucial roles in modulating saccade velocity, especially in the ODR task. What was striking was that the answering saccades had the highest peak velocity and the shortest saccade duration comparing to error categories as if the marmosets knew whether they would answer correctly or not. This may be reflecting the confidence of the marmosets in making the saccades (Seideman et al., 2018).

Finally, we strongly recommend maintaining a high motivation level for the marmosets during behavior training. Because if they experienced continuous episodes of failure and no reward, they lose their enthusiasm and eventually give up on doing the task. In agreement with that, previous macaque monkey study has shown that when monkeys were given the choice to do or abandon a task with high difficulty, they choose to opt out of the task (Smith et al., 1998).

\section{Conflict of Interest}

The authors declare that the research was conducted in the absence of any commercial or financial relationships that could be construed as a potential conflict of interest.

\section{Author Contributions}

WA was responsible for the behavioural training, data collection, formal analysis, and the writing of the first draft. CC was involved in guiding the research and editing the manuscript. $\mathrm{HO}$ was involved in the marmosets' MRI imaging and surgeries. The project was overseen and supervised by TI. All authors shaped the final version of the manuscript and approved the submitted version.

\section{$7 \quad$ Funding}

This research was supported by AMED under Grant Number JP19dm0207093 to H.O. and T.I.; JSPS Grant-in-Aid for Research Activity Start-up Grant Number JP20K22684 to C.Y.C.

\section{Acknowledgments}

This manuscript is published in the non-peer reviewed preprint server, bioRxiv, under doi: https://doi.org/10.1101/2021.08.29.458139 (Amly et al., 2021). 
Amly, W., Chen, C. ., Onoe, H., and Isa, T. (2021). Dissecting errors of the exogenously-driven and endogenously-driven saccadic tasks in the common marmosets. bioRxiv. doi:doi.org/10.1101/2021.08.29.458139.

Antoniades, C. A., and Kennard, C. (2015). Ocular motor abnormalities in neurodegenerative disorders. Eye 29, 200-207. doi:10.1038/eye.2014.276.

Badler, J. B., and Heinen, S. J. (2006). Anticipatory movement timing using prediction and external cues. J. Neurosci. 26, 4519-4525. doi:10.1523/JNEUROSCI.3739-05.2006.

Bahill, A. T., Clark, M. R., and Stark, L. (1975). The main sequence, a tool for studying human eye movements. Math. Biosci. 24, 191-204. doi:10.1016/0025-5564(75)90075-9.

Bellet, M. E., Bellet, J., Nienborg, H., Hafed, Z. M., and Berens, P. (2019). Human-level saccade detection performance using deep neural networks. J. Neurophysiol. 121, 646-661. doi:10.1152/jn.00601.2018.

Benedek, M., Stoiser, R., Walcher, S., and Körner, C. (2017). Eye behavior associated with internally versus externally directed cognition. Front. Psychol. 8, 1-9. doi:10.3389/fpsyg.2017.01092.

Bittencourt, J., Velasques, B., Teixeira, S., Basile, L. F., Salles, J. I., Nardi, A. E., et al. (2013). Saccadic eye movement applications for psychiatric disorders. Neuropsychiatr. Dis. Treat. 9, 1393-1409. doi:10.2147/NDT.S45931.

Carney, H. C., Hart, E., and Huk, A. C. (2019). Demonstration and quantification of memory-guided saccades in the common marmoset (with comparison to the macaque). J. Vis. 19, 86a.

569 doi:10.1167/19.10.86a.

Chen, C.-Y., Matrov, D., Veale, R., Onoe, H., Yoshida, M., Miura, K., et al. (2021). Properties of visually guided saccadic behavior and bottom-up attention in marmoset, macaque, and human. J. Neurophysiol. 125, 437-457. doi:10.1152/jn.00312.2020.

Chen, L. L., Hung, L. Y., Quinet, J., and Kosek, K. (2013). Cognitive regulation of saccadic velocity by reward prospect. Eur. J. Neurosci. 38, 2434-2444. doi:10.1111/ejn.12247.

Di Stasi, L. L., Antolí, A., and Cañas, J. J. (2011). Main sequence: An index for detecting mental

Clarke, H. F., Hill, G. J., Robbins, T. W., and Roberts, A. C. (2011). Dopamine, but not serotonin, regulates reversal learning in the marmoset caudate nucleus. J. Neurosci. 31, 4290-4297. doi:10.1523/JNEUROSCI.5066-10.2011. workload variation in complex tasks. Appl. Ergon. 42, 807-813. doi:10.1016/j.apergo.2011.01.003.

Emborg, M. E. (2017). Nonhuman primate models of neurodegenerative disorders. ILAR J. 58, 190201. doi:10.1093/ilar/ilx021.

Eslamboli, A., Romero-Ramos, M., Burger, C., Bjorklund, T., Muzyczka, N., Mandel, R. J., et al. (2007). Long-term consequences of human alpha-synuclein overexpression in the primate 
ventral midbrain. Brain 130, 799-815. doi:10.1093/brain/awl382.

Fox, S. H., Visanji, N., Reyes, G., Huot, P., Gomez-Ramirez, J., Johnston, T., et al. (2010). Neuropsychiatric behaviors in the MPTP marmoset model of Parkinson's disease. Can. J. Neurol. Sci. 37, 86-95. doi:10.1017/S0317167100009707.

Fuchs, A. F. (1967). Saccadic and smooth pursuit eye movements in the monkey. J. Physiol. 191, 609-631. doi:10.1113/jphysiol.1967.sp008271.

592

593

Fukushima, K., Fukushima, J., and Barnes, G. R. (2017). Clinical application of eye movement tasks as an aid to understanding Parkinson's disease pathophysiology. Exp. Brain Res. 235, 13091321. doi:10.1007/s00221-017-4916-5.

Funahashi, S., Bruce, C. J., and Goldman-Rakic, P. S. (1989). Mnemonic coding of visual space in the monkey's dorsolateral prefrontal cortex. J. Neurophysiol. 61, 331-349.

597 doi:10.1152/jn.1989.61.2.331.

Gnadt, J. W., and Andersen, R. A. (1988). Memory related motor planning activity in posterior parietal cortex of macaque. Exp. brain Res. 70, 216-20. doi:10.1007/BF00271862.

Gnadt, J. W., Bracewell, R. M., and Andersen, R. A. (1991). Sensorimotor transformation during eye movements to remembered visual targets. Vision Res. 31, 693-715. doi:10.1016/0042-

601 6989(91)90010-3.

603

Goffart, L., Quinet, J., Chavane, F., and Masson, G. S. (2006). Influence of background illumination

604

605 on fixation and visually guided saccades in the rhesus monkey. Vision Res. 46, 149-162. doi:10.1016/j.visres.2005.07.026.

Goldstone, R. L. (1998). PERCEPTUAL LEARNING. Annu. Rev. Psychol. 49, 585-612. doi:10.1146/annurev.psych.49.1.585.

Gooding, D. C., and Basso, M. A. (2008). The tell-tale tasks: A review of saccadic research in

Harris, C. M. (1995). Does saccadic undershoot minimize saccadic flight-time? A Monte-Carlo study. Vision Res. 35, 691-701. doi:10.1016/0042-6989(94)00163-G.

Harris, C. M., and Wolpert, D. M. (2006). The main sequence of saccades optimizes speed-accuracy trade-off. Biol. Cybern. 95, 21-29. doi:10.1007/s00422-006-0064-х.

Heeman, J., Van der Stigchel, S., Munoz, D. P., and Theeuwes, J. (2019). Discriminating between anticipatory and visually triggered saccades: Measuring minimal visual saccadic response time using luminance. J. Neurophysiol. 121, 2101-2111. doi:10.1152/jn.00378.2018.

Hikosaka, O., and Wurtz, R. H. (1983). Visual and oculomotor functions of monkey substantia nigra pars reticulata. I. Relation of visual and auditory responses to saccades. J. Neurophysiol. 49, 1230-1253. doi:10.1152/jn.1983.49.5.1230. 
doi:10.1016/j.neuron.2015.03.021.

Jendritza, P., Klein, F. J., Rohenkohl, G., and Fries, P. (2021). Visual neuroscience methods for marmosets: Efficient receptive field mapping and head-free eye tracking. eNeuro 8.

624 doi:10.1523/ENEURO.0489-20.2021.

Jenner, P., Rupniak, N. M. J., Rose, S., Kelly, E., Kilpatrick, G., Lees, A., et al. (1984). 1-Methyl-4phenyl-1,2,3,6-tetrahydropyridine-induced parkinsonism in the common marmoset. Neurosci. Lett. 50, 85-90. doi:10.1016/0304-3940(84)90467-1.

Johnston, K. D., Barker, K., Schaeffer, L., Schaeffer, D., and Everling, S. (2018). Methods for chair restraint and training of the common marmoset on oculomotor tasks. J. Neurophysiol. 119, 1636-1646. doi:10.1152/jn.00866.2017.

Johnston, K., Ma, L., Schaeffer, L., and Everling, S. (2019). Alpha oscillations modulate preparatory activity in marmoset area 8Ad. J. Neurosci. 39, 1855-1866. doi:10.1523/JNEUROSCI.270318.2019.

Kapoula, Z. (1985). Evidence for a Range Effect Saccadic System. Vision Res. 25, 1155-1157. doi:/doi.org/10.1016/0042-6989(85)90105-1.

Kuvaldina, M., Chetverikov, A. A., Odainic, A. S., Filippova, M. G., and Andriyanova, N. V. (2019). "Implicit learning from one's mistakes," in Implicit Learning (Routledge), 108-132.

639 doi:10.4324/9781315628905-5.

Lebedev, S., Van Gelder, P., and Wai Hon Tsui (1996). Square-root relations between main saccadic parameters. Investig. Ophthalmol. Vis. Sci. 37, 2750-2758.

Leigh, R. J., and Kennard, C. (2004). Using saccades as a research tool in the clinical neurosciences. Brain 127, 460-477. doi:10.1093/brain/awh035.

Lin, M. K., Takahashi, Y. S., Huo, B. X., Hanada, M., Nagashima, J., Hata, J., et al. (2019). A highthroughput neurohistological pipeline for brain-wide mesoscale connectivity mapping of the common marmoset. Elife 8, 1-36. doi:10.7554/eLife.40042.

Mitchell, J. F., Reynolds, J. H., and Miller, C. T. (2014). Active vision in marmosets: A model system for visual neuroscience. J. Neurosci. 34, 1183-1194. doi:10.1523/JNEUROSCI.389913.2014 . saccadic peak velocity associated with individual differences in motivation. Cortex 122, 198212. doi:10.1016/j.cortex.2018.12.001. the infant and adult common marmoset. Nat. Protoc. 11, 1299-1308. doi:10.1038/nprot.2016.076. 
659

660

661

662

663

664

665

666

667

668

669

670

671

672

673

674

675

676

677

678

679

680

681

682

683

684

685

686

687

688

689

690

691

692

693

694

Nuthmann, A., Vitu, F., Engbert, R., and Kliegl, R. (2016). No evidence for a saccadic range effect for visually guided and memory-guided Saccades in simple saccade-targeting tasks. PLoS One 11, 1-27. doi:10.1371/journal.pone.0162449.

Okano, H. (2021). Current Status of and Perspectives on the Application of Marmosets in Neurobiology. Annu. Rev. Neurosci. 44, 27-48. doi:10.1146/annurev-neuro-030520-101844.

Opris, I., Barborica, A., and Ferrera, V. P. (2003). Comparison of performance on memory-guided saccade and delayed spatial match-to-sample tasks in monkeys. Vision Res. 43, 321-332. doi:10.1016/S0042-6989(02)00418-2.

Opwonya, J., Doan, D. N. T., Kim, S. G., Kim, J. Il, Ku, B., Kim, S., et al. (2021). Saccadic Eye Movement in Mild Cognitive Impairment and Alzheimer's Disease: A Systematic Review and Meta-Analysis. doi:10.1007/s11065-021-09495-3.

Park, J. E., Zhang, X. F., Choi, S. H., Okahara, J., Sasaki, E., and Silva, A. C. (2016). Generation of transgenic marmosets expressing genetically encoded calcium indicators. Sci. Rep. 6, 1-12. doi:10.1038/srep34931.

Posner, M. I. (1980). Orienting of attention. Q. J. Exp. Psychol. 32, 3-25. doi:10.1080/00335558008248231.

Powers, A. S., Basso, M. A., and Evinger, C. (2013). Blinks slow memory-guided saccades. J. Neurophysiol. 109, 734-741. doi:10.1152/jn.00746.2012.

Pratt, J., Bekkering, H., Abrams, R. A., and Adam, J. (1999). The Gap effect for spatially oriented responses. Acta Psychol. (Amst). 102, 1-12. doi:10.1016/S0001-6918(99)00014-1.

Qiu, Z., and Li, X. (2017). Non-human Primate Models for Brain Disorders - Towards Genetic Manipulations via Innovative Technology. Neurosci. Bull. 33, 247-250. doi:10.1007/s12264017-0115-4.

Ramat, S., Leigh, R. J., Zee, D. S., and Optican, L. M. (2007). What clinical disorders tell us about the neural control of saccadic eye movements. Brain 130, 10-35. doi:10.1093/brain/awl309.

Reichle, E. D., Reineberg, A. E., and Schooler, J. W. (2010). Eye movements during mindless reading. Psychol. Sci. 21, 1300-1310. doi:10.1177/0956797610378686.

Reulen, J. P. H. (1984). Latency of visually evoked saccadic eye movements - II. Temporal properties of the facilitation mechanism. Biol. Cybern. 50, 263-271. doi:10.1007/BF00337076.

Sasaki, E., Suemizu, H., Shimada, A., Hanazawa, K., Oiwa, R., Kamioka, M., et al. (2009). Generation of transgenic non-human primates with germline transmission. Nature 459, 523527. doi:10.1038/nature08090.

Saslow, M. G. (1967). Latency for saccadic eye movement. J. Opt. Soc. Am. 57, 1030-1033. doi:10.1364/JOSA.57.001030.

Sedaghat-Nejad, E., Herzfeld, D. J., Hage, P., Karbasi, K., Palin, T., Wang, X., et al. (2019). Behavioral training of marmosets and electrophysiological recording from the cerebellum. $J$. 
Neurophysiol. 122, 1502-1517. doi:10.1152/jn.00389.2019.

696

697

698

699

700

701

702

703

704

705

706

707

708

709

710

711

712

713

714

715

716

717

718

719

720

721

722

723

724

725

726

727

728

729

730
Seideman, J. A., Stanford, T. R., and Salinas, E. (2018). Saccade metrics reflect decision-making dynamics during urgent choices. Nat. Commun. 9. doi:10.1038/s41467-018-05319-w.

Smallwood, J., McSpadden, M., and Schooler, J. W. (2007). The lights are on but no one's home: Meta-awareness and the decoupling of attention when the mind wanders. Psychon. Bull. Rev. 14, 527-533. doi:10.3758/BF03194102.

Smith, J. D., Shields, W. E., Allendoerfer, K. R., and Washburn, D. A. (1998). Memory monitoring by animals and humans. J. Exp. Psychol. Gen. 127, 227-250. doi:10.1037/0096-3445.127.3.227.

Spinelli, S., Pennanen, L., Dettling, A. C., Feldon, J., Higgins, G. A., and Pryce, C. R. (2004). Performance of the marmoset monkey on computerized tasks of attention and working memory. Cogn. Brain Res. 19, 123-137. doi:10.1016/j.cogbrainres.2003.11.007.

Stanford, T. R., and Sparks, D. L. (1994). Systematic errors for saccades to remembered targets: Evidence for a dissociation between saccade metrics and activity in the superior colliculus. Vision Res. 34, 93-106. doi:10.1016/0042-6989(94)90260-7.

Tam, W. J., and Ono, H. (1994). Fixation disengagement and eye-movement latency. Percept. Psychophys. 56, 251-260. doi:10.3758/BF03209759.

Tardif, S. D., Mansfield, K. G., Ratnam, R., Ross, C. N., and Ziegler, T. E. (2011). The Marmoset as a Model of Aging and Age-Related Diseases. ILAR J. 52, 54-65. doi:10.1093/ilar.52.1.54.

Termsarasab, P., Thammongkolchai, T., Rucker, J. C., and Frucht, S. J. (2015). The diagnostic value of saccades in movement disorder patients: a practical guide and review. J. Clin. Mov. Disord. 2, 1-10. doi:10.1186/s40734-015-0025-4.

Tomioka, I., Ishibashi, H., Minakawa, E. N., Motohashi, H. H., Takayama, O., Saito, Y., et al. (2017). Transgenic monkey model of the polyglutamine diseases recapitulating progressive neurological symptoms. eNeuro 4, 1-16. doi:10.1523/ENEURO.0250-16.2017.

Verdier, J. M., Acquatella, I., Lautier, C., Devau, G., Trouche, S., Lasbleiz, C., et al. (2015). Lessons from the analysis of nonhuman primates for understanding human aging and neurodegenerative diseases. Front. Neurosci. 9, 1-6. doi:10.3389/fnins.2015.00064.

Vermilyea, S. C., Guthrie, S., Meyer, M., Smuga-Otto, K., Braun, K., Howden, S., et al. (2017). Induced Pluripotent Stem Cell-Derived Dopaminergic Neurons from Adult Common Marmoset Fibroblasts. Stem Cells Dev. 26, 1225-1235. doi:10.1089/scd.2017.0069.

Waldthaler, J., Stock, L., Student, J., Sommerkorn, J., Dowiasch, S., and Timmermann, L. (2021). Antisaccades in Parkinson's Disease: A Meta-Analysis. Neuropsychol. Rev. doi:10.1007/s11065-021-09489-1.

White, J. M., Sparks, D. L., and Stanford, T. R. (1994). Saccades to remembered target locations: an analysis of systematic and variable errors. Vision Res. 34, 79-92. doi:10.1016/00426989(94)90259-3. 
731

732

733

734

Yamazaki, Y., Echigo, C., Saiki, M., Inada, M., Watanabe, S., and Iriki, A. (2011). Tool-use learning by common marmosets (Callithrix jacchus). Exp. Brain Res. 213, 63-71. doi:10.1007/s00221011-2778-9.

Yarbus, A. L. (1967). "Eye Movements During Perception of Complex Objects," in Eye Movements and Vision (Boston, MA: Springer US), 171-211. doi:978-1-4899-5379-7.

Zee, D. S., and Leigh, R. J. (1983). Disorders of Eye Movements. Neurol. Clin. 1, 909-928. doi:10.1016/S0733-8619(18)31131-9.

\section{Data Availability Statement}

Data presented in this paper are available upon reasonable request.

\section{$11 \quad$ Figure legends}

Figure 1 Task illustration. (A) Gap task. A 1-degree-diameter marmoset face was used as both fixation stimulus and target stimulus. (B) Oculomotor delayed response (ODR) task. A 1-degreediameter white dot with black surrounding was used as both fixation stimulus and target stimulus. The 2-deg-diameter eye window was indicated with a dashed circle in each task. Marmoset's gaze fixation was indicated as a red arrowhead and the saccade that they made to acquire the reward was indicated as a blue arrow.

Figure 2 Change in success rate over the training period. (A) Gap task. The success rate for each session was plotted in relation to the cumulative training sessions, separated by the gap period. The data here was platted regardless of the target eccentricity. We selected $0 \mathrm{~ms}$ (left column), $150 \mathrm{~ms}$ (middle column), and $300 \mathrm{~ms}$ (right column) as examples. (B) Similar plots were generated for the ODR task. We selected $150 \mathrm{~ms}$ (left column) and $300 \mathrm{~ms}$ (right column) as examples. The black line in each plot is the moving average of the success rate with a window size of 10 sessions and moving at 1 session step.

Figure 3 Radial distance between the target location and the saccade. (A) Definition of first saccades. We analyzed the radial distance of the first saccade after target onset in gap task or fixation offset in ODR task regardless of whether the saccade vector was toward the target or not, from the successful trials. (B) Radial distance between the target location in the gap task and the first saccade. 4-degree and 6-degree target eccentricities are plotted here as examples from all marmosets. An example of undershooting (hypometric) saccade with less than 2-degree is indicated by an arrowhead. (C) Radial distance between the target location in the ODR task and the first saccade. 4degree and 6-degree target eccentricities are plotted here as examples for all marmosets. An example of overshooting (hypermetric) saccade with radial distance more than 2-degree is indicated by an arrowhead. (D) Definition of answering saccades. We further constrained the saccades with start point and endpoint all within the eye window and defined them as answering saccades (also see Figure 4A). (E) Radial distance between the target location in the gap task and the answering saccade. (F) Radial distance between the target location in the ODR task and the answering saccade. The median of the radial distance is indicated in each figure.

Figure 4 Subgroups from the successful trials. (A) Definition of the answering saccade and subgroups from the successful trials. By definition, answering saccades are found only in trials of 
pure single and single group, not in the multiple group. (B) Percentage of trials in each subgroup from the successful trials. Note that in the ODR task, the pure single group was higher in both marmoset $\mathrm{S}$ and marmoset $\mathrm{M}$ than in the gap task. The multiple groups have a lower percentage of trials in the ODR task than in the gap task for all marmosets.

775 Figure 5 Saccade response time (SRT) from the successful trials of gap and ODR task. Only answering saccades were included for SRT calculation. (A) Averaged SRT of gap task. Note that the SRT for all marmosets continued to decrease for the gap period 0 to $150 \mathrm{~ms}$. (B) Averaged SRT of ODR task. No consistent SRT change was observed across the delay period. Data shown here were collected regardless of the target eccentricity. Shaded areas around the colored lines indicate the standard error mean (S.E.M.) of the SRT. The black solid line in each plot indicates the mean SRT from the three marmosets.

782

Figure 6 Description of the error categories in the gap and ODR tasks. (A) Definition of error categories in the gap task. Error trials consist of three types of error, defined according to when the trial was aborted. The eye position shown here represents a visuomotor error trial with saccade response time (SRT) extracted. If the SRT was less than $70 \mathrm{~ms}$, the trial was relabeled as an impulsive error trial and the SRT would be recalculated based on the second nearest monitor event (in this case, fixation stimulus offset). The black dashed lines around the eye position indicate eye windowing for monitoring the marmosets' eye position. (B) Example saccades extracted in each error category from the gap task. Here we plotted randomly selected error trials from marmoset $\mathrm{S}$ for each error category. For comparison, we also plotted randomly selected successful trials from the same marmoset. Dashed circles indicate the eye windowing size and locations of potential target stimuli. (C) Definition of error categories in the ODR task. A similar convention is used as in (A). (D) Example of saccades extracted from each error category from the ODR task. Here we plotted randomly selected trials from marmoset $\mathrm{S}$ for each category. A similar convention is used as in (B).

Figure 7 Percentage of trials in each category of gap and ODR task. After separating the error trials into proper error categories (distractive, impulsive, or visuomotor), we plotted the percentage of each category including successful trials collected from the beginning of training regardless of target eccentricity or gap/delay period. (A) Gap task. (B) ODR task.

799 Figure 8 Saccade response time (SRT) from all categories of gap and ODR task. SRTs were 800 plotted for each error and successful trials regardless of target eccentricity or gap/delay period for either gap or ODR task. (A) SRT of distractive error category. Zero in the x-axis indicates fixation stimulus onset time for both tasks. (B) SRT of impulsive error category. Zero in the x-axis indicates fixation stimulus offset time in the gap and target stimulus onset in ODR task. (C) SRT of visuomotor error category. Zero in the $\mathrm{x}$-axis indicates target stimulus onset time in gap and fixation stimulus offset time in ODR task. (D) SRT of successful category. Zero in the x-axis indicates target stimulus onset time in gap and fixation stimulus offset time in ODR task. Similar to Figure 5, only answering saccade was used to calculate SRT. The median of SRT for each task is indicated in the

809 Figure 9 Current error saccade and previous decision in the ODR task. (A) Saccades direction of 810 the current error trials. We separated the saccades vector and calculated if the saccade direction was 811 toward the target location or away from it. (B) Reasons for failure of saccade toward the target in 812 visuomotor trials. We found most of the saccades were undershooting saccades and did not reach the target eye window. A small portion of the saccades was overshooting. Another small portion of the 814 saccades was that the marmoset did not stay enough time in the target eye window. (C) Previous trial 
815 type and saccade direction relative to current saccade direction. We took only visuomotor error trials

816 with saccade away from the target in this analysis. We found a similar percentage of the same and the opposite saccade direction comparing previous to current trial if the previous trial was a successful

818 trial. However, if the previous trial was an impulsive trial, the marmosets tend to switch their saccade

819 direction. If the previous trial was also a visuomotor trial, the marmosets tend to keep the same

820 saccade direction.

821 Figure 10 Comparing saccade main sequence across error and successful categories. (A)

822 Saccade amplitude against saccade peak velocity. We plotted saccades extracted from each error and

823 successful trials for gap task. Fitting parameters are: Marmoset S: distractive: $a=84.6, n=0.817$;

824 impulsive: $\mathrm{a}=64.08, \mathrm{n}=0.967$; visuomotor: $\mathrm{a}=48.98, \mathrm{n}=1.135$; successful: $\mathrm{a}=118, \mathrm{n}=0.768$;

825

826

827 Marmoset N: distractive: $a=81.45, n=0.84$; impulsive: $a=67.58, n=0.94$; visuomotor: $a=57.85, n=$ 1.003; successful: $\mathrm{a}=105, \mathrm{n}=0.854$; Marmoset M: distractive: $\mathrm{a}=80.68, \mathrm{n}=0.897$; impulsive: $\mathrm{a}=$ 73.85, $\mathrm{n}=$ 0.955; visuomotor: $\mathrm{a}=58.47, \mathrm{n}=1.096$; successful: $\mathrm{a}=123.8, \mathrm{n}=0.789$. (B) Saccade amplitude against saccade peak velocity. Here we plotted saccades extracted from each error and successful trials for ODR task. Fitting parameters are: Marmoset S: distractive: $a=42.54, n=1.13$; impulsive: $a=89.57, n=0.9077$; visuomotor: $a=66.9, n=0.978$; successful: $a=80.11, n=0.989$; Marmoset N: impulsive: $a=106, n=0.756$; visuomotor: $a=58.97, n=0.974$; successful: $a=53.07, n=$ 1.21; Marmoset M: distractive: $\mathrm{a}=92.12, \mathrm{n}=0.872$; impulsive: $\mathrm{a}=108.3, \mathrm{n}=0.851$; visuomotor: $\mathrm{a}=$ 81.34, $n=0.904$; successful: $a=89.45, n=0.984$. (C) Saccade amplitude against saccade duration. Here we plotted saccades extracted from each error and successful trials for gap task. Fitting parameters are: Marmoset $S$ : distractive: $b=2.47, c=23.24$; impulsive: $b=1.67, c=29.76$; visuomotor: $b=1.448, c=31.39$; successful: $b=1.73, c=20.7$; Marmoset $N$ : distractive: $b=2.33, c=$ 24.09; impulsive: $b=1.77, c=27.17$; visuomotor: $b=1.68, c=30.1$; successful: $b=1.09, c=22.3$;

838 Marmoset M: distractive: $b=2.05, c=26.57$; impulsive: $b=1.63, c=28.03$; visuomotor: $b=1.19, c=$ 30.39; successful: $b=1.31$, $c=20.6$. (D) Saccade amplitude against saccade duration. Here we plotted saccades extracted from each error and successful trials for ODR task. Fitting parameters are: Marmoset S: distractive: $b=2.83, c=28.69$; impulsive: $b=0.86, c=26.56$; visuomotor: $b=1.63, c=$ 29.26; successful: $b=0.39, c=28.59$; Marmoset $N$ : impulsive: $b=2.19, c=21.36$; visuomotor: $b=$ 2.09, $c=29.20$; successful: $b=-0.52, c=34.69$; Marmoset $M$ : distractive: $b=2.17, c=29.25$; impulsive: $b=1.44, c=21.97$; visuomotor: $b=1.96, c=27.05$; successful: $b=0.37, c=26.82$. Solid curves indicate the fitted curve. 
A

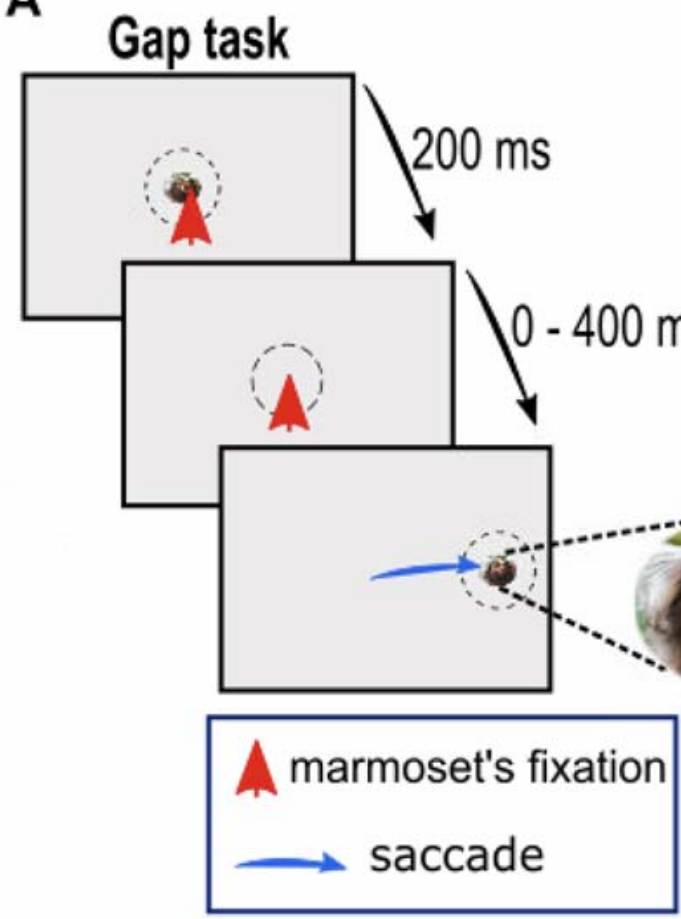

B

\section{ODR task}

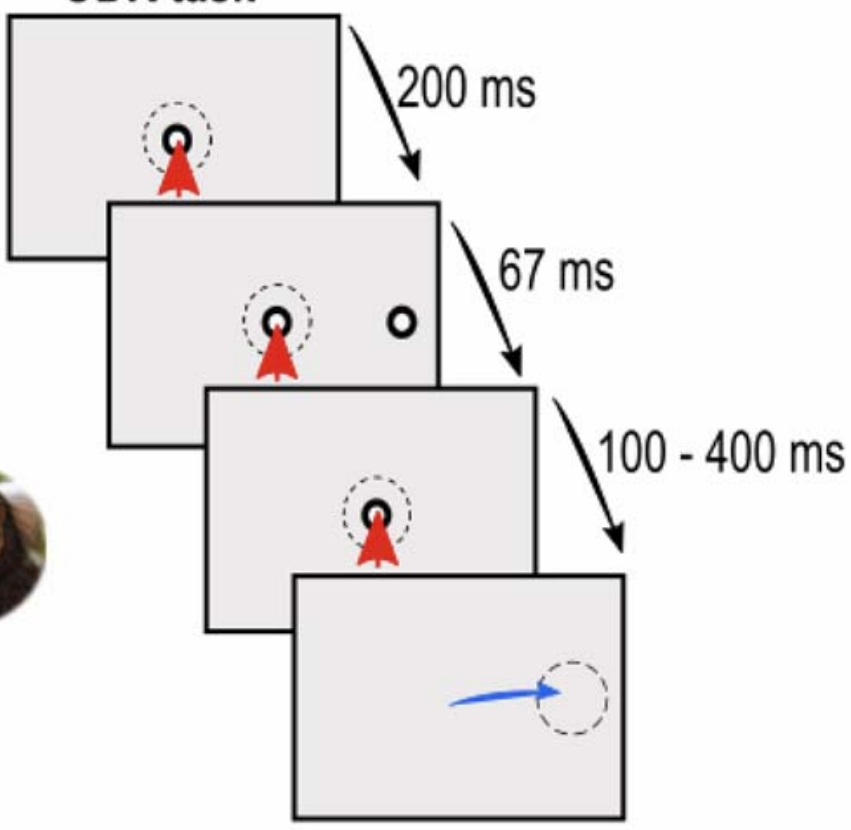


A
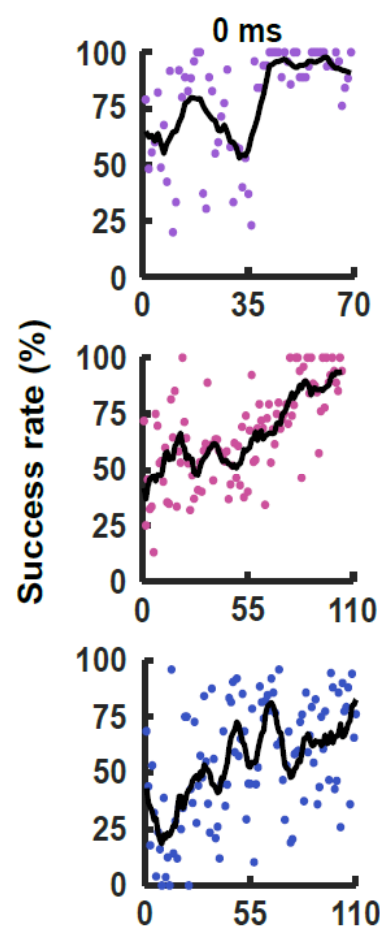

849
Gap
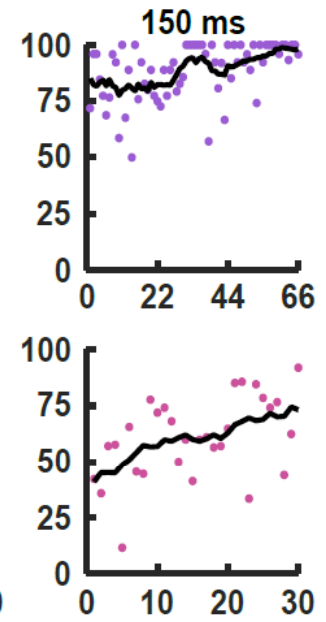

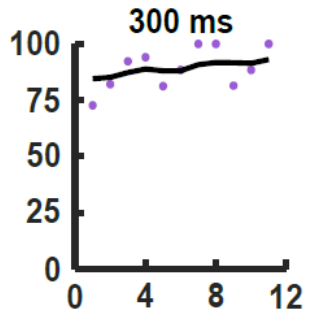

B

ODR
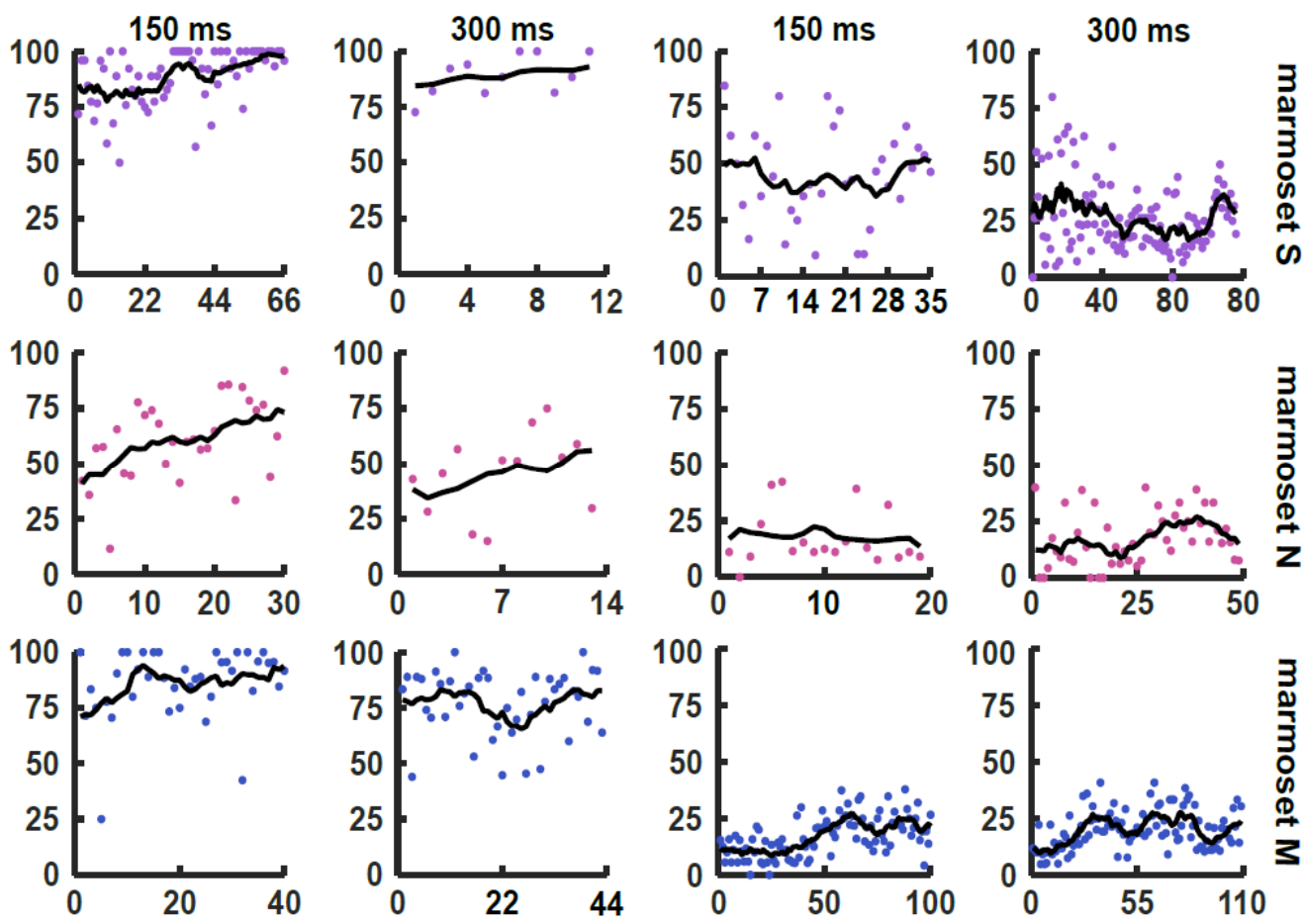

Cumulative training sessions

\section{$850 \quad$ Figure 2}



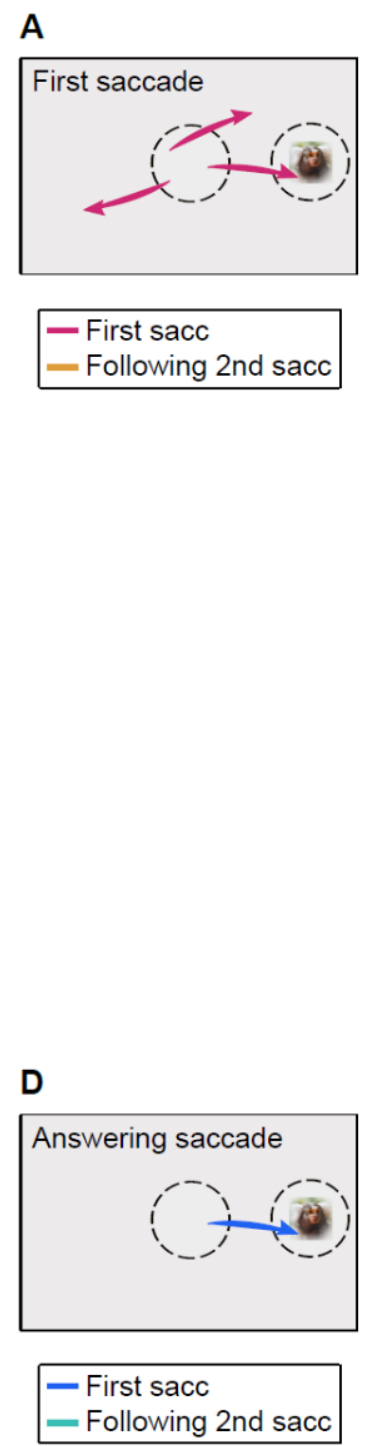

852

853

Figure 3

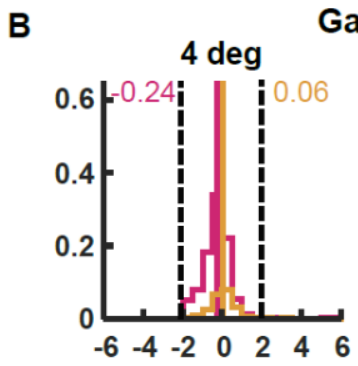

Gap $6 \mathrm{deg}$

C 4 deg ODR
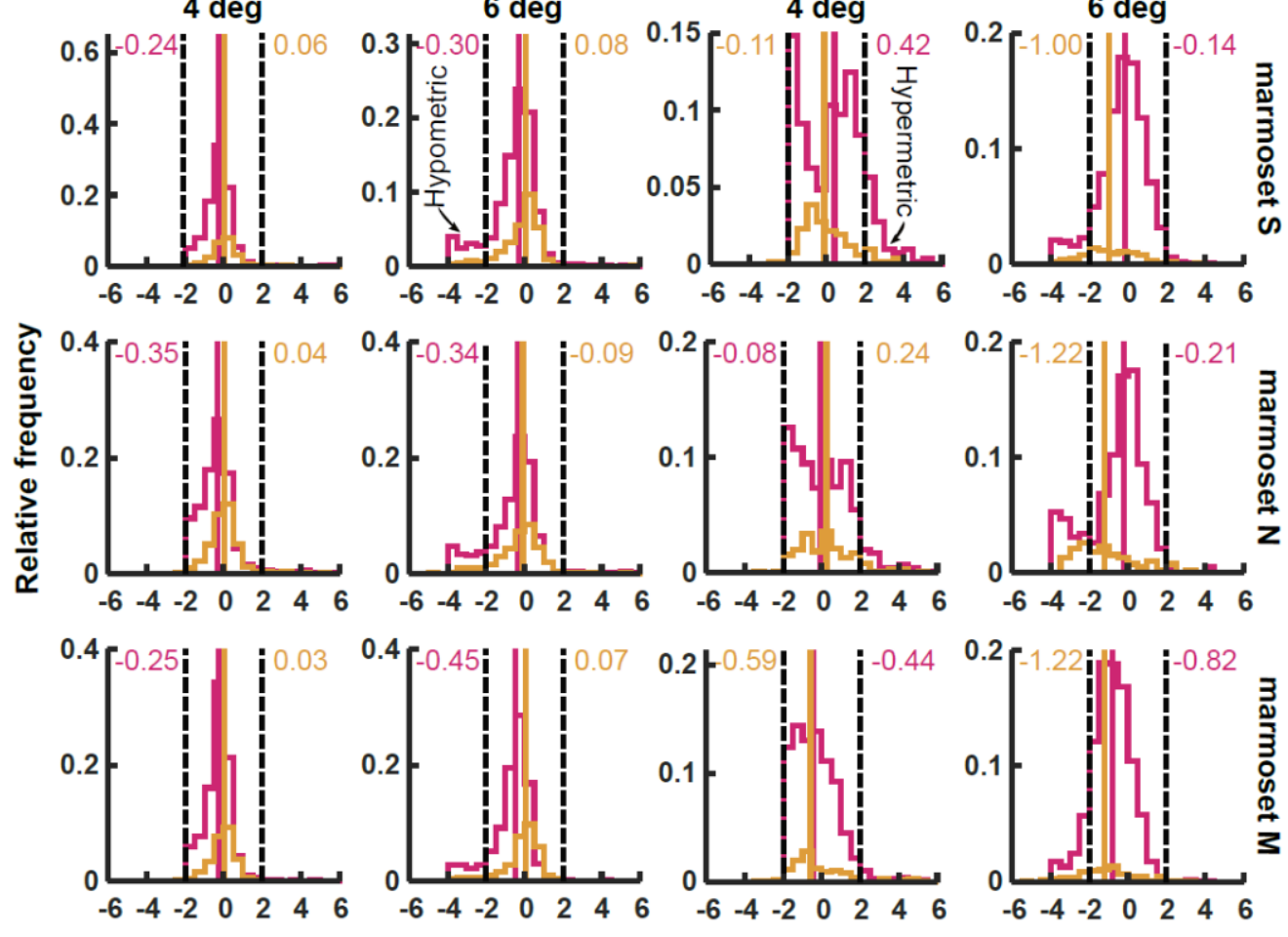

Radial distance from the target (deg)

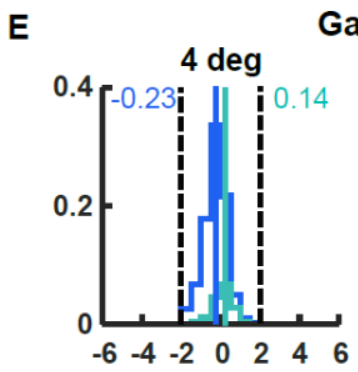

Gap

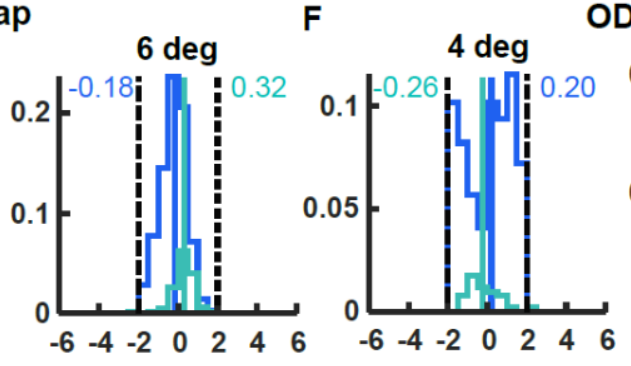

ODR
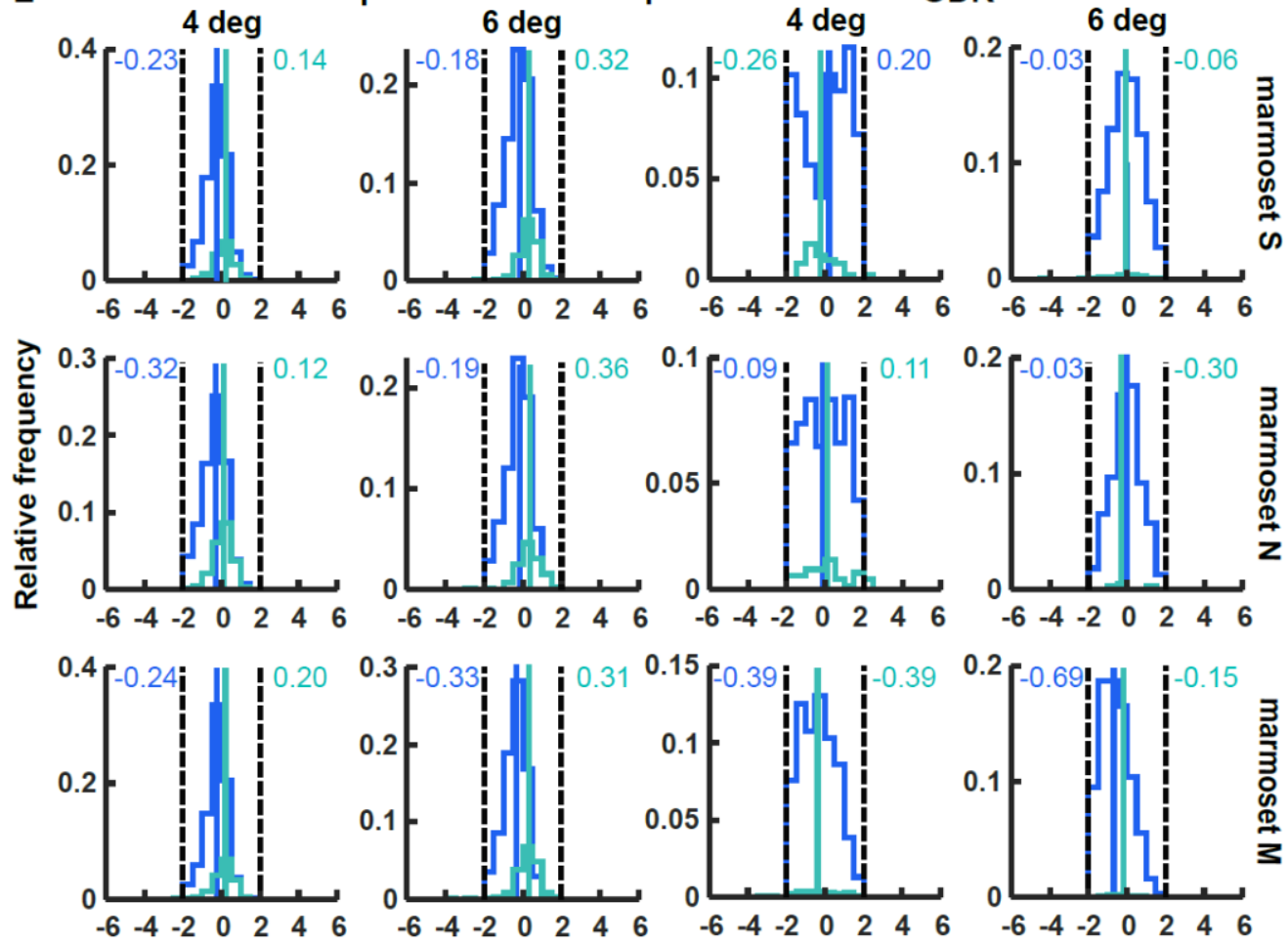

Radial distance from the target (deg) 
bioRxiv preprint doi: https://doi.org/10.1101/2021.08.29.458139- this version posted September 2, 2021. The copyright holder for this preprint (which was not certified by peer review) is the author/funder, who has granted bioRxiv a license to display the preprint in perpetuity. It is made available under aCC-BY-NC-ND 4.0 International license.

A

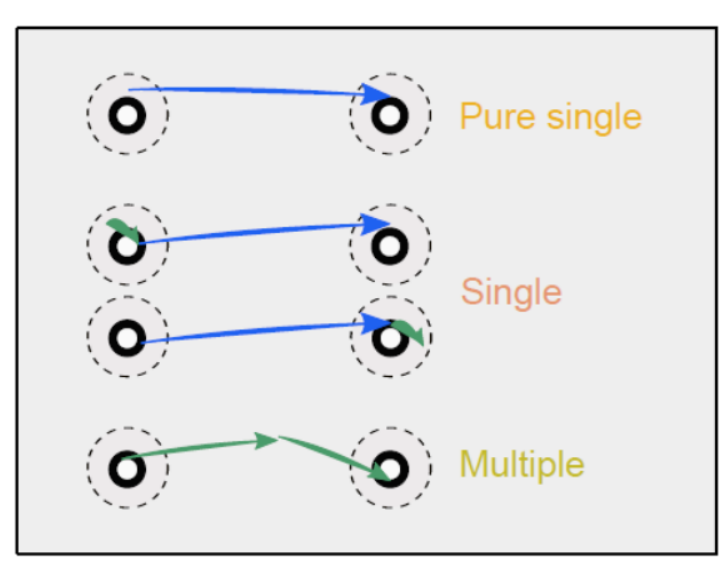

855

\section{$856 \quad$ Figure 4}

857
Pure single

Single

Multiple

Answering

Non-answering
B
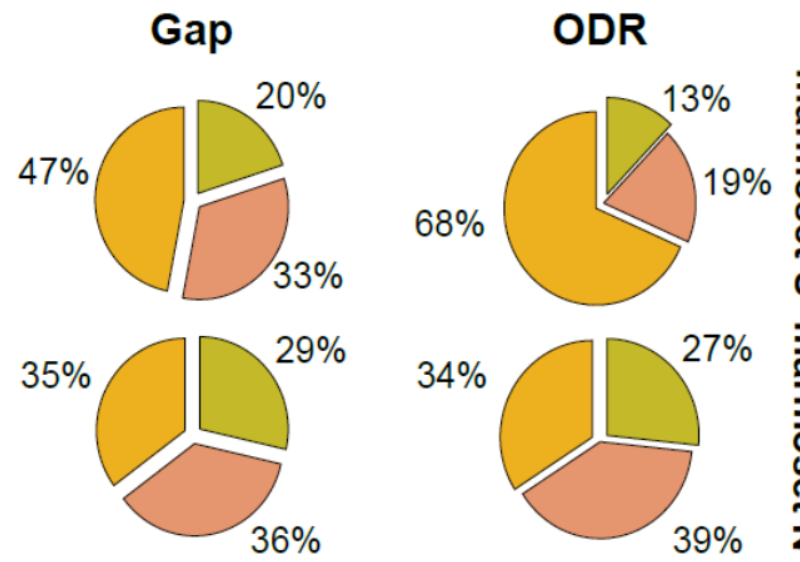

בె๊

$34 \%$

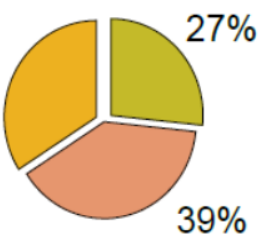

ฏ
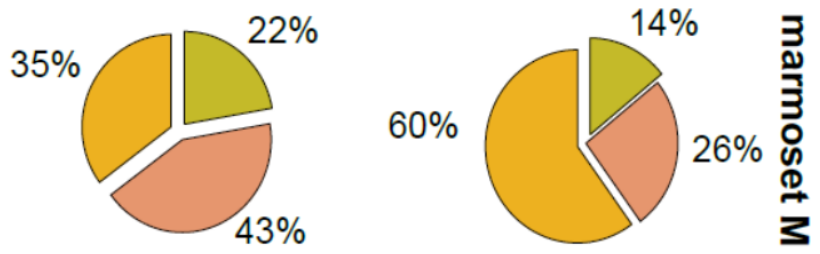
bioRxiv preprint doi: https://doi.org/10.1101/2021.08.29.458139; this version posted September 2, 2021. The copyright holder for this preprint (which was not certified by peer review) is the author/funder, who has granted bioRxiv a license to display the preprint in perpetuity. It is made available under aCC-BY-NC-ND 4.0 International license.
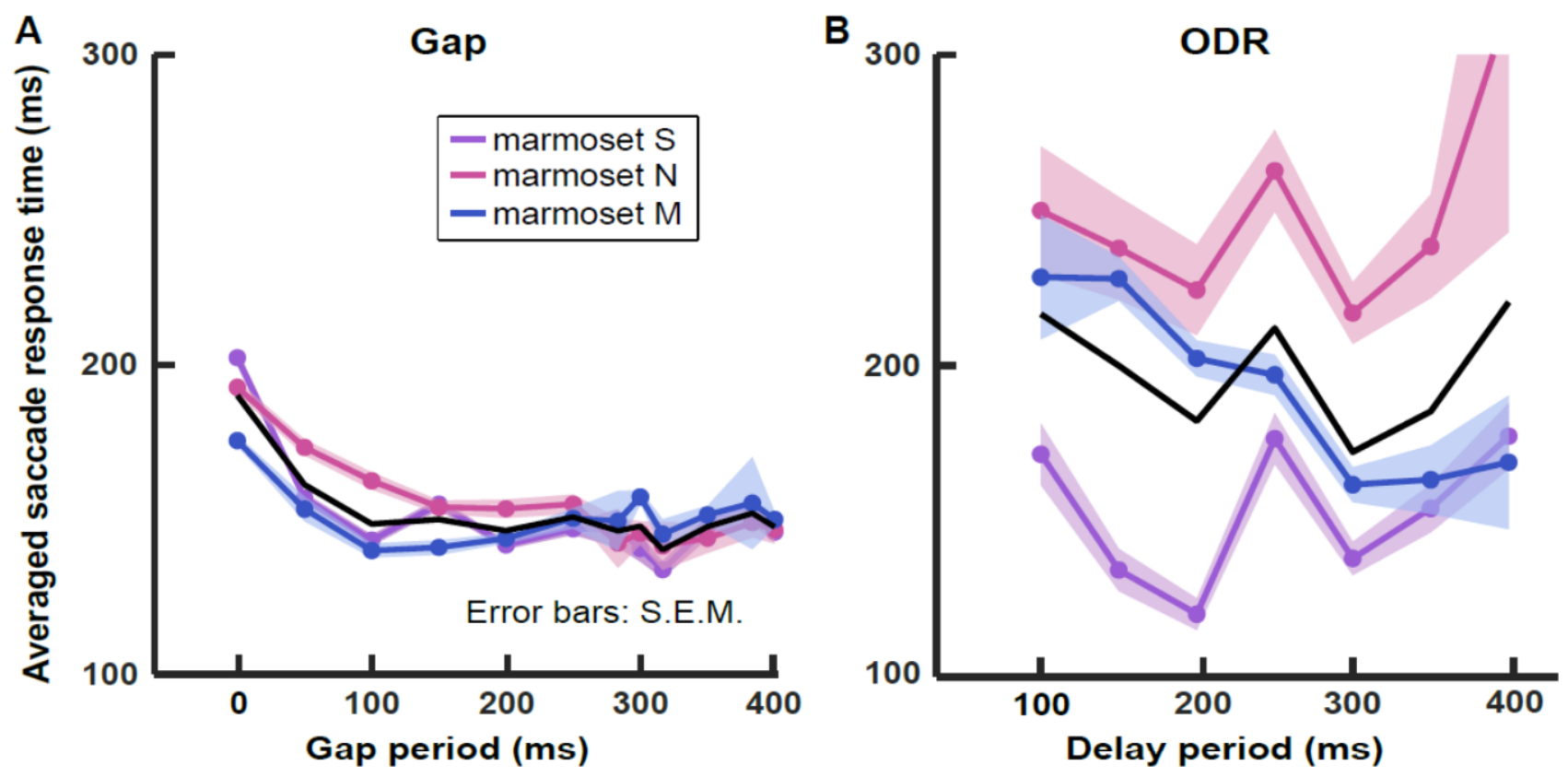

859 Figure 5

860 
A Gap
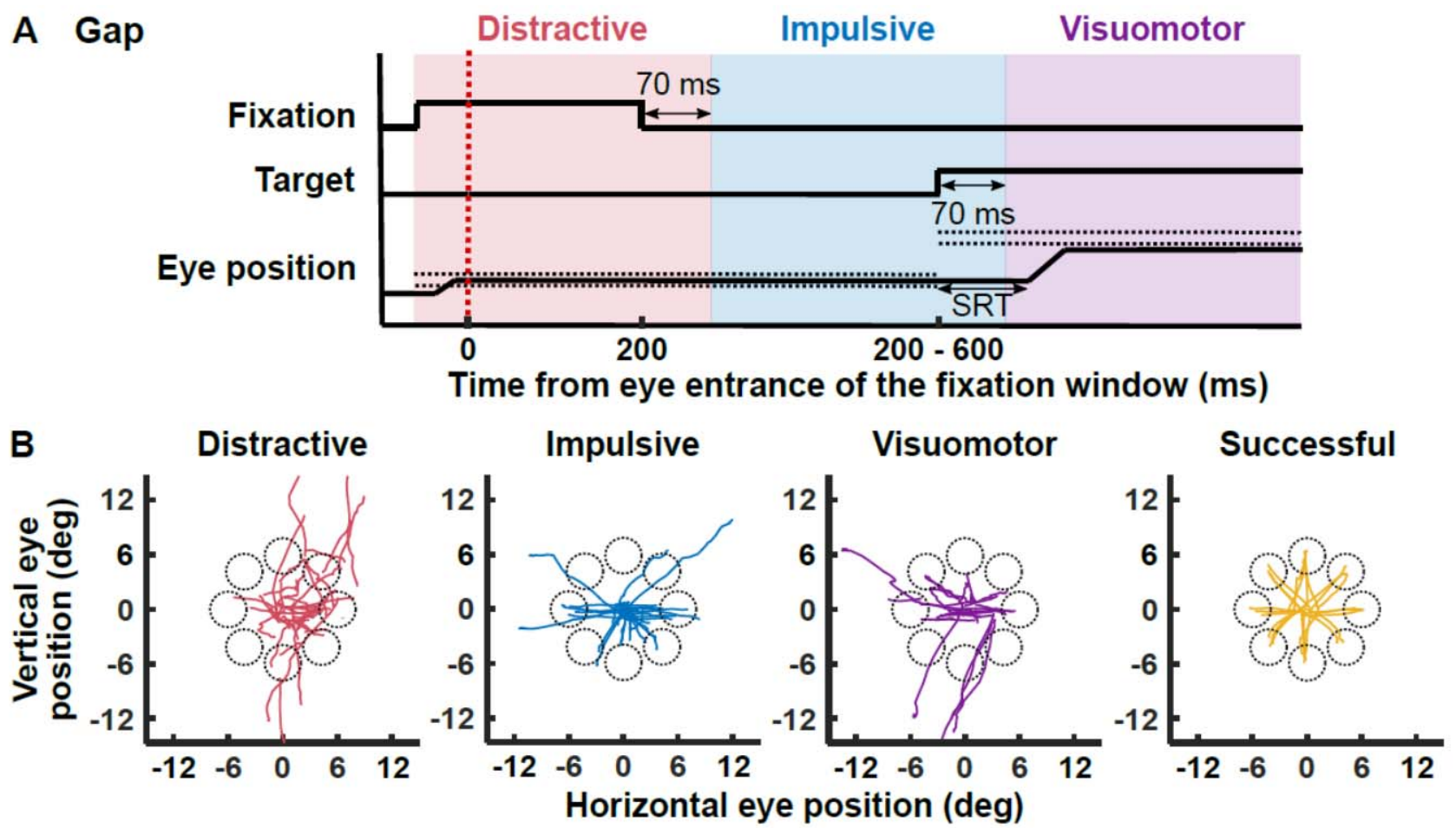

C ODR
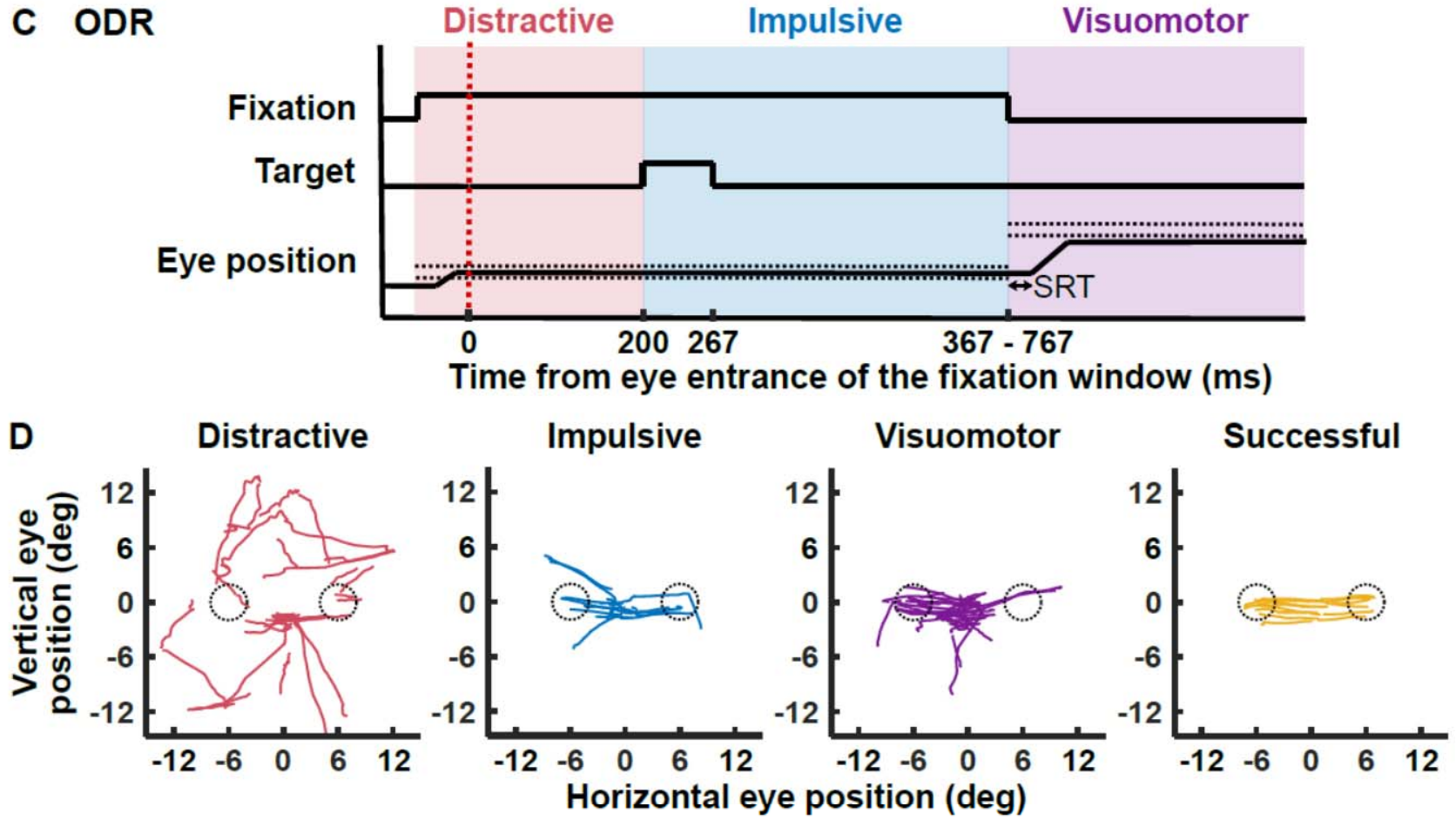

$862 \quad$ Figure 6 
bioRxiv preprint doi: https://doi.org/10.1101/2021.08.29.458139; this version posted September 2, 2021. The copyright holder for this preprint (which was not certified by peer review) is the author/funder, who has granted bioRxiv a license to display the preprint in perpetuity. It is made available under aCC-BY-NC-ND 4.0 International license.

A Gap
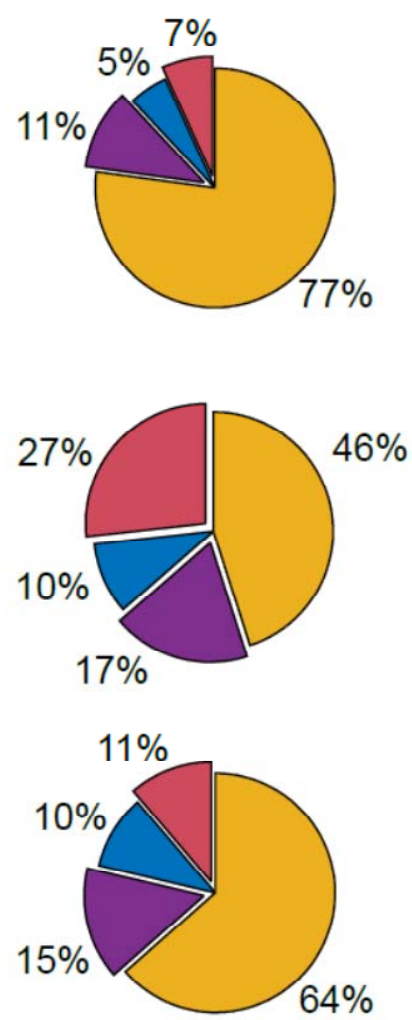

B ODR

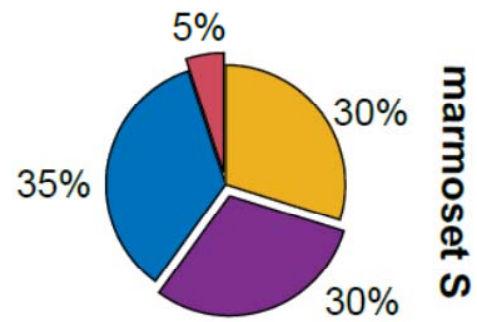

$31 \%$

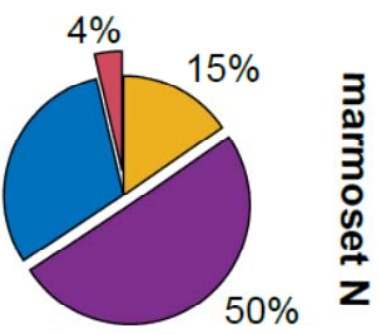

$38 \%$

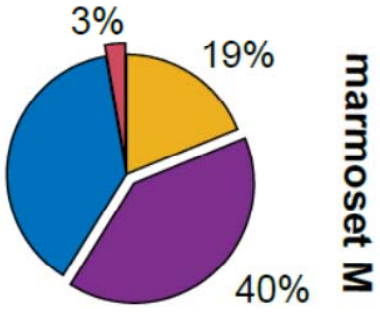

864

$\begin{array}{ll}\text { Distractive } & \text { Visuomotor } \\ \text { Impulsive } & \text { Successful }\end{array}$

\section{$865 \quad$ Figure 7}

866 


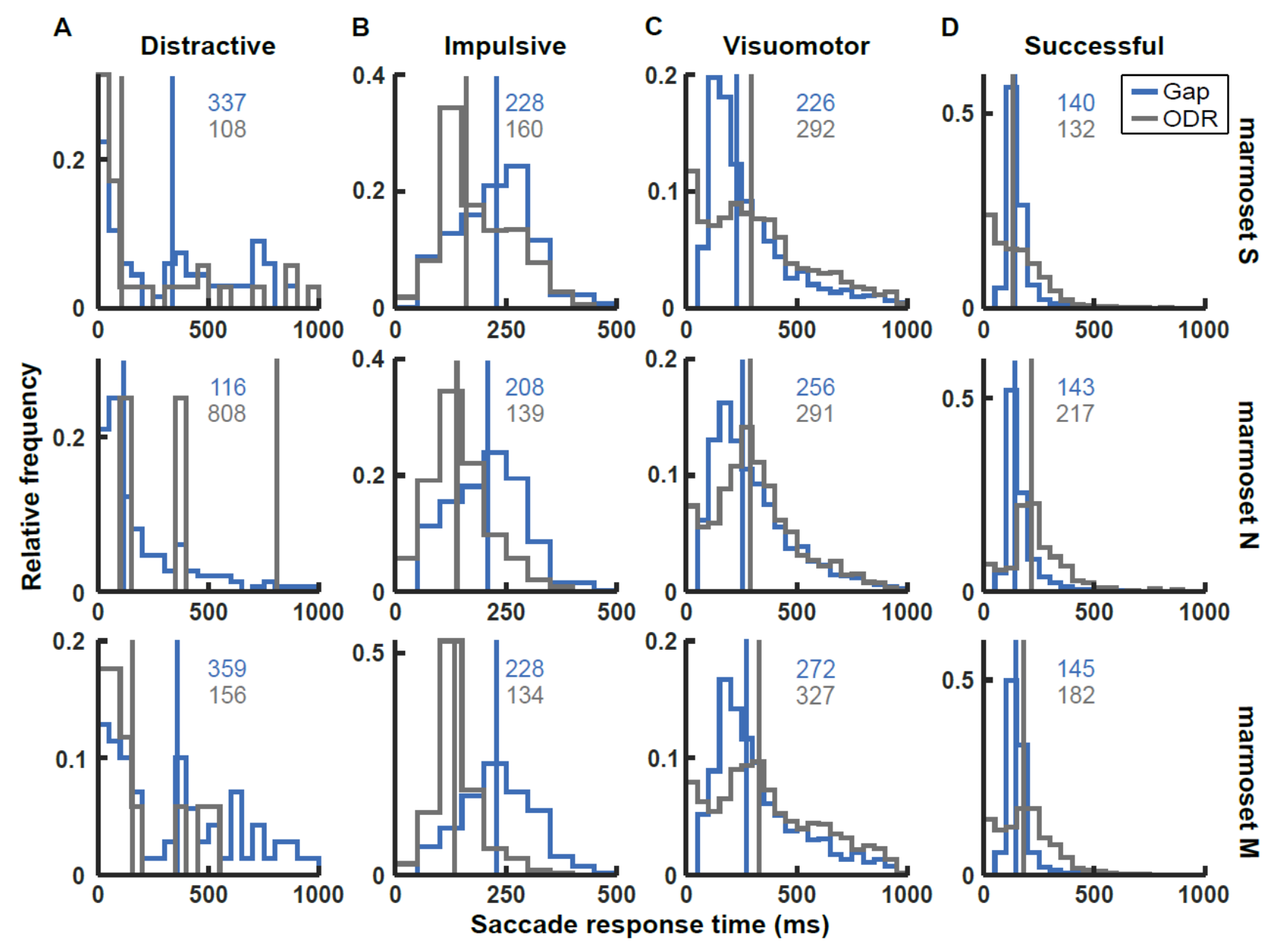

$868 \quad$ Figure 8

869 

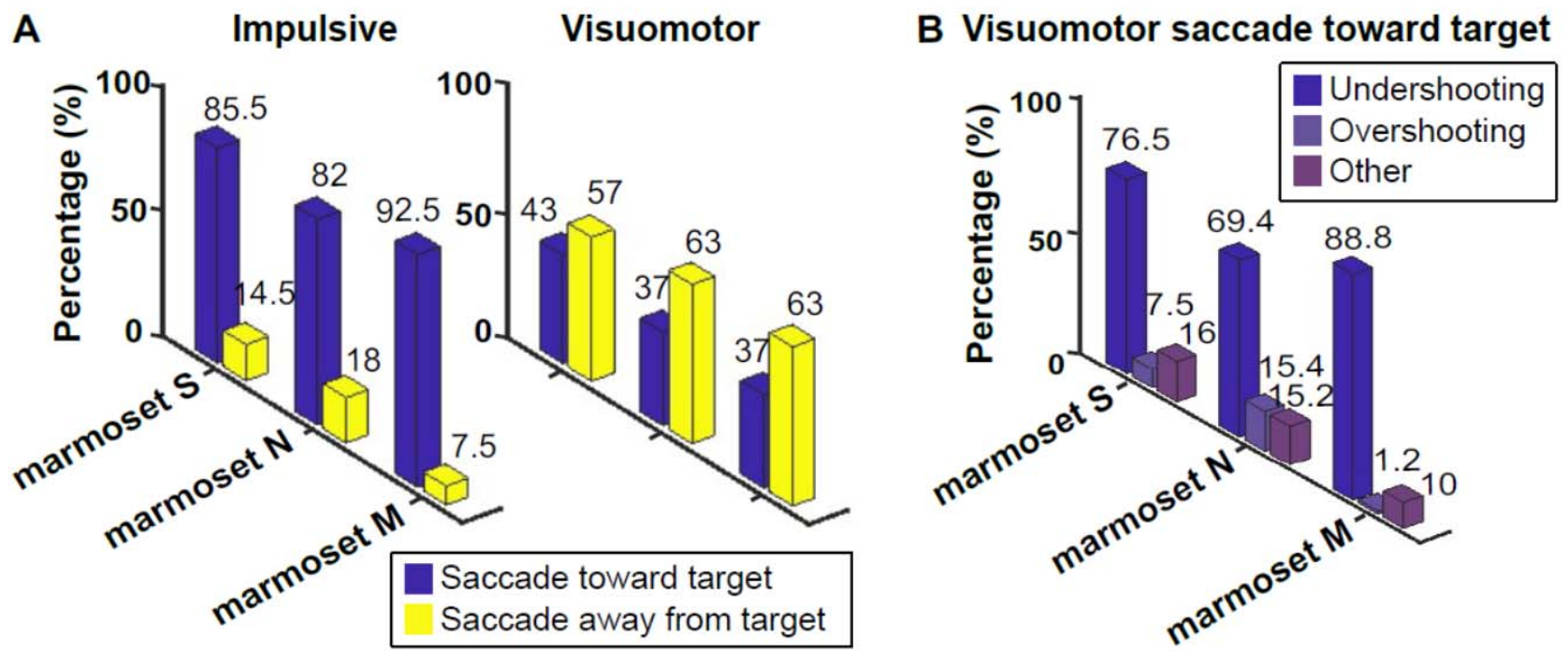

C Successful $\rightarrow$ Visuomotor Impulsive $\rightarrow$ Visuomotor Visuomotor $\rightarrow$ Visuomotor

870

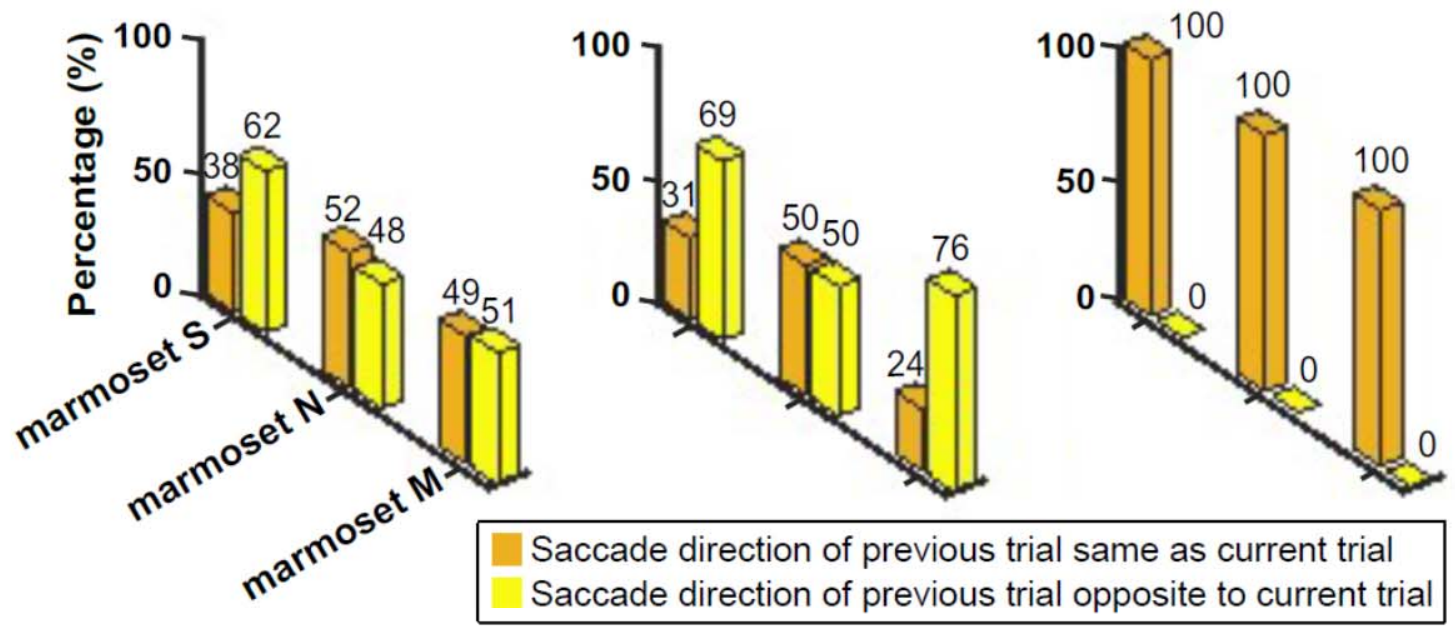

$871 \quad$ Figure 9

872 
bioRxiv preprint doi: https:/doi org/10.1101/2021.08.29.458139. this version posted September 2, 2021. The copyright holder for this preprint (which was not certified by peer review) is the author/funder, who has granted bioRxiv a license to display the preprint in perpetuity. It is made available under aCC-BY-NC-ND 4.0 International license.

A
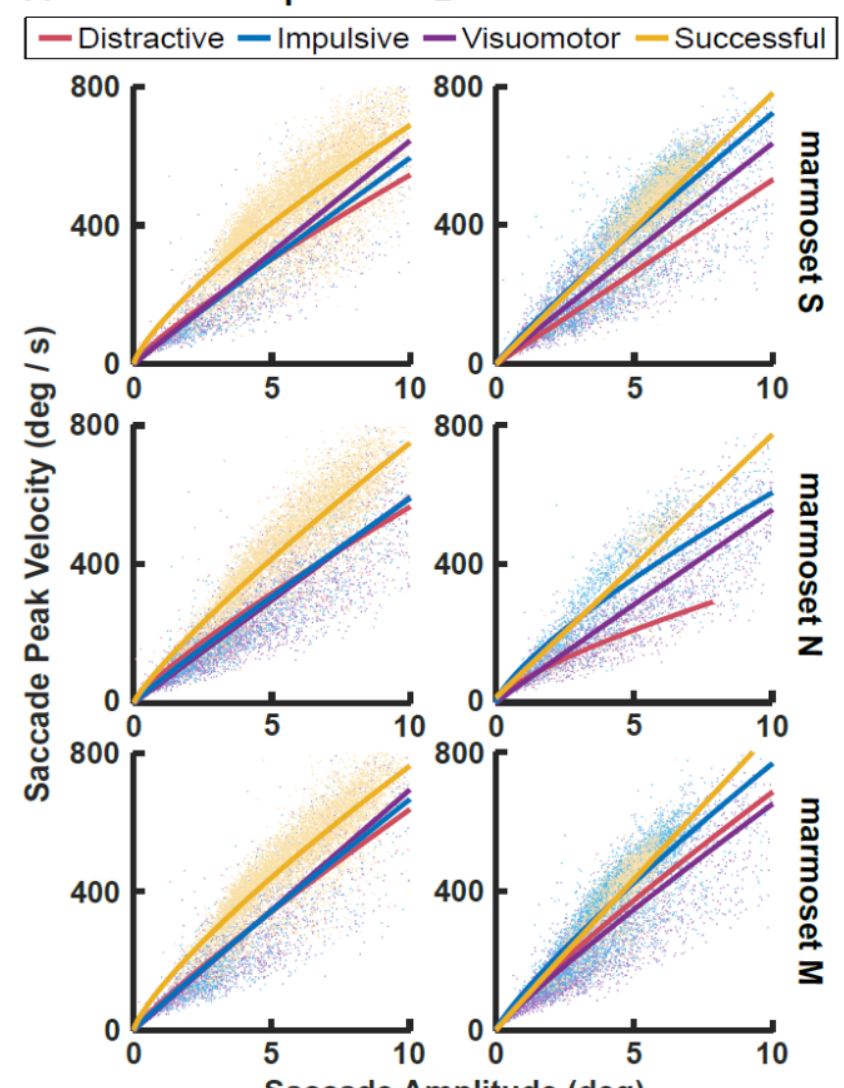

800
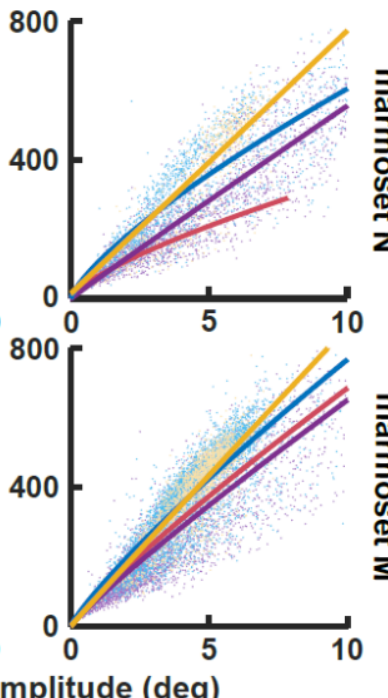

873

C

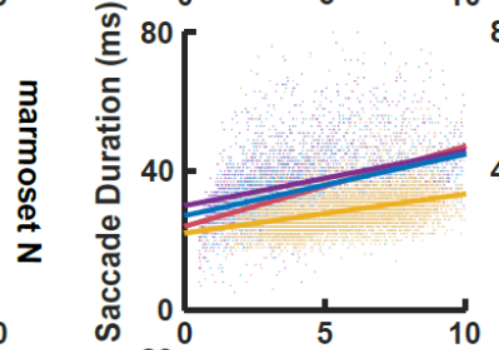

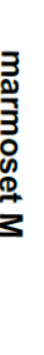

Gap D ODR
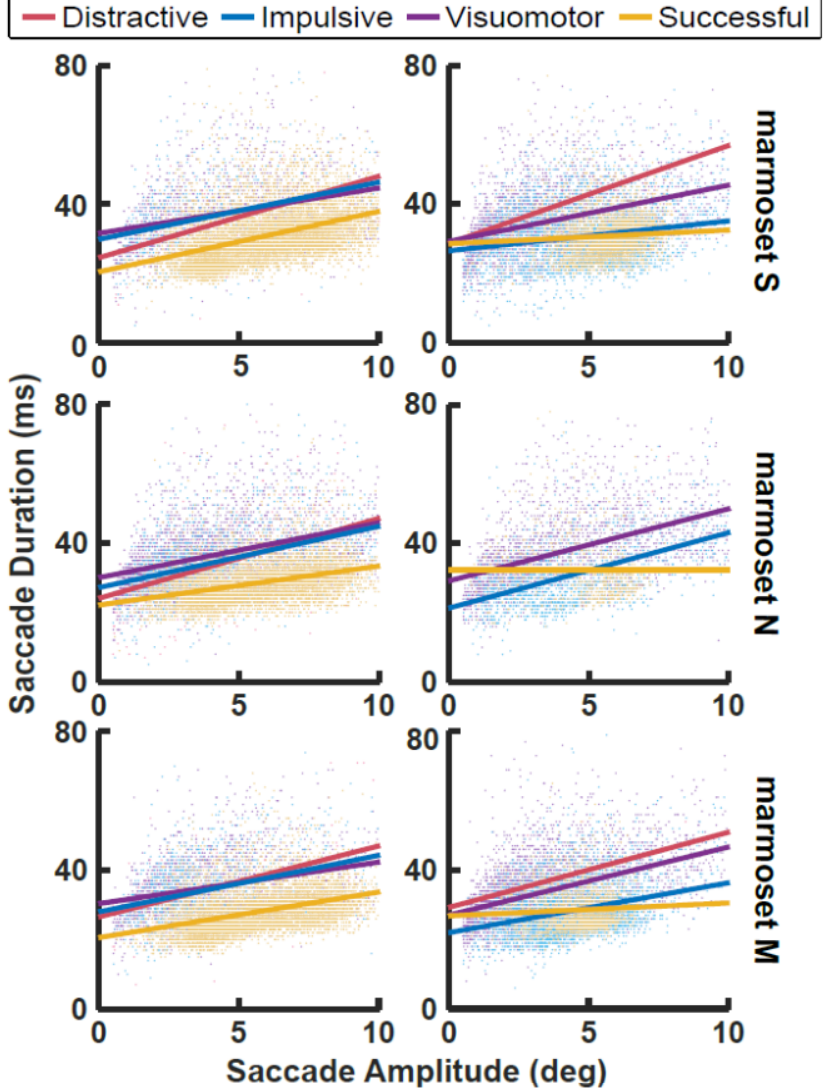

\section{Figure 10}

875 
A
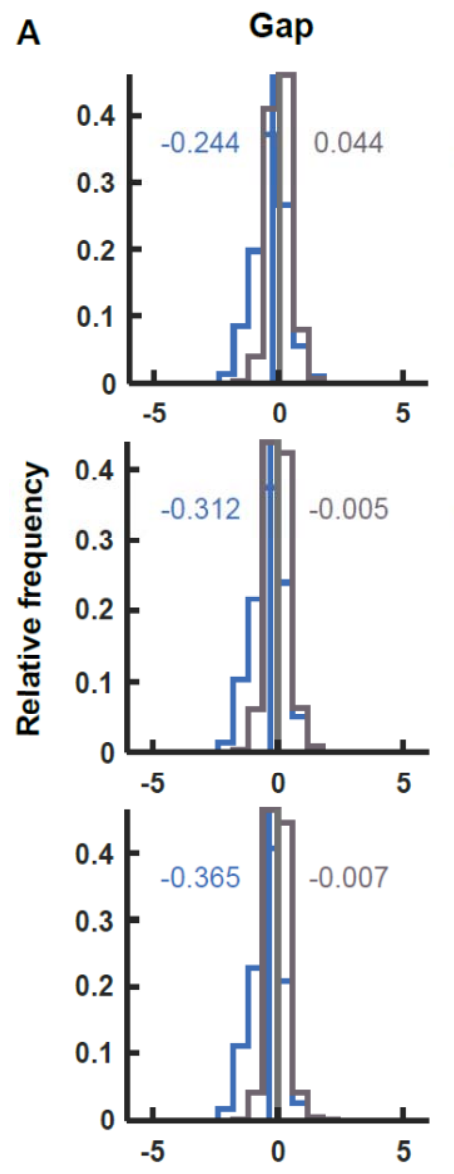
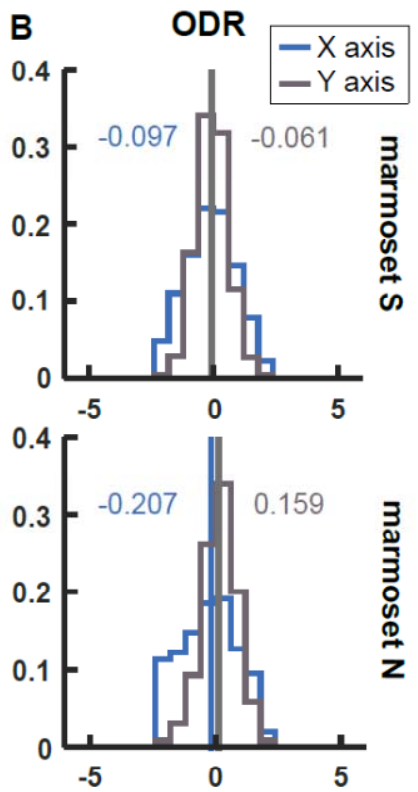

877

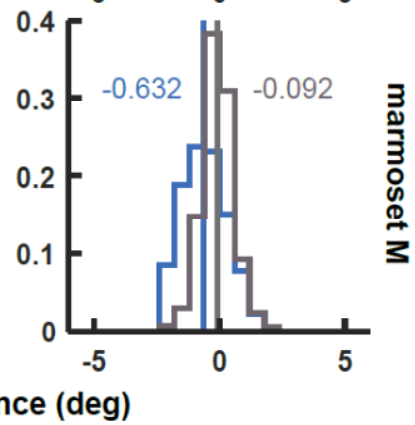

878 Supplementary Figure 1 Distance from the target location to answering saccade endpoint. In this figure, we combined all the answering saccades from successful trials regardless of the gap or delay period, or target eccentricity. Previously, an upward bias of the saccade trajectory and landing position was reported in both exogenously-driven and endogenously-driven saccadic tasks (White et al., 1994; Goffart et al., 2006). To preserve possible upward shift from our data set, we first flipped the target locations in the second and the third quadrant and the correspondent answering saccade along the $\mathrm{x}$-axis. We then rotated all the target locations and the answering saccades to the positive $\mathrm{x}$ axis. Finally, we took the difference between the target and the $\mathrm{x}$ and $\mathrm{y}$ coordinates of the saccade endpoint independently. (A) Result from gap task. (B) The result from ODR task. As can be seen, the undershooting on the $\mathrm{x}$ distance from the saccade endpoint to the target location was always more than the y distance for both tasks. (Gap: marmoset $\mathrm{S}: \mathrm{p}<2.2 \times 10^{-308}$, marmoset $\mathrm{N}: \mathrm{p}=3.4 \times 10^{-251}$, marmoset $\mathrm{M}, \mathrm{p}<2.2 \times 10^{-308}$; ODR: marmoset $\mathrm{S}: \mathrm{p}=9.9 \times 10^{-3}$, marmoset $\mathrm{N}: \mathrm{p}=3.1 \times 10^{-14}$, marmoset $\mathrm{M}, \mathrm{p}=2.4 \times 10^{-96}$; Wilcoxon rank sum test) and there was no systematic upward or downward bias in 891 the y distance. 
A

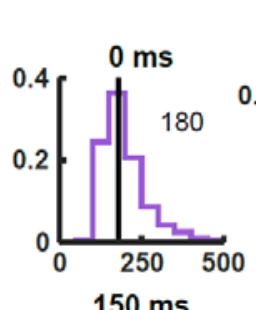

Gap
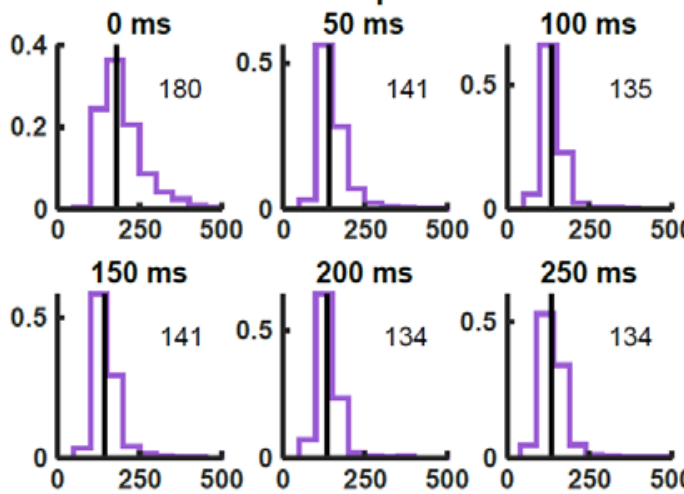

$200 \mathrm{~ms}$
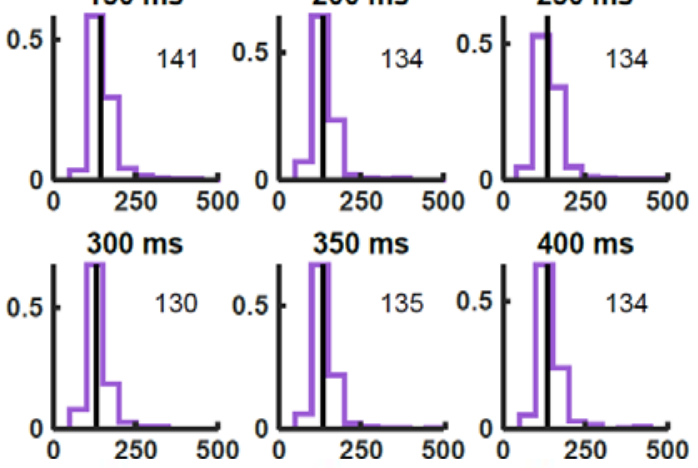

$400 \mathrm{~ms}$
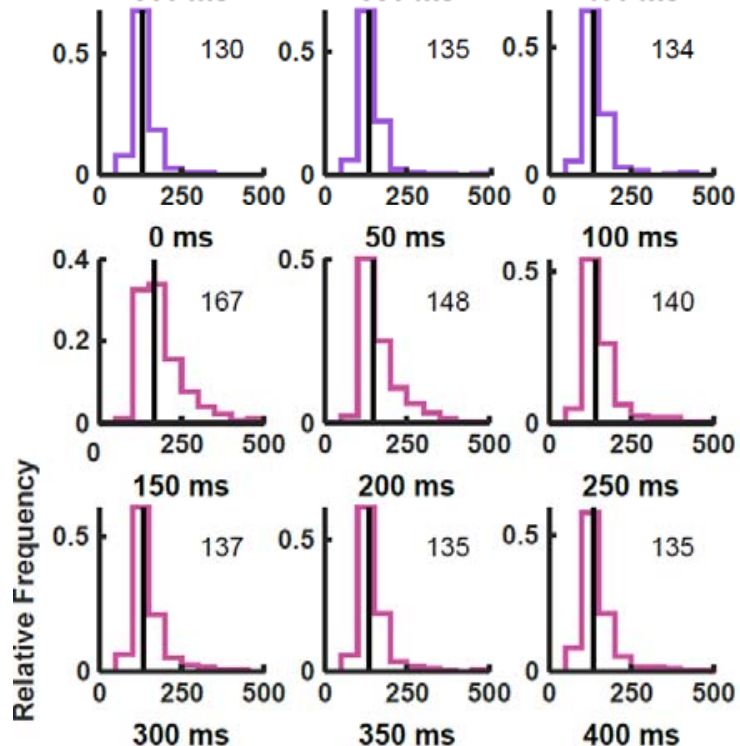

$200 \mathrm{~ms}$
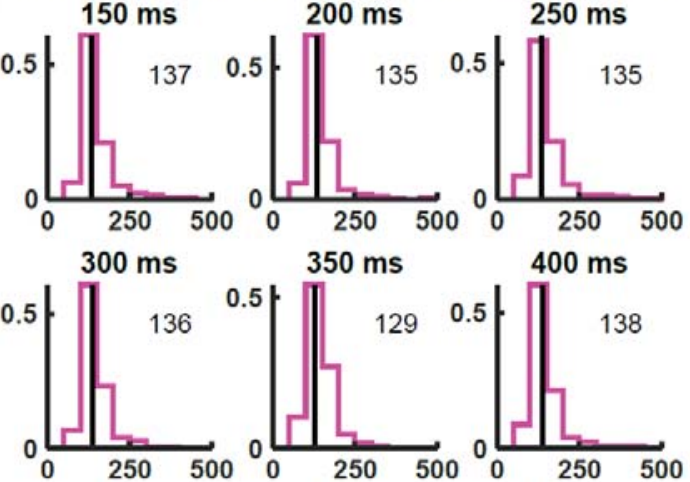

$400 \mathrm{~ms}$
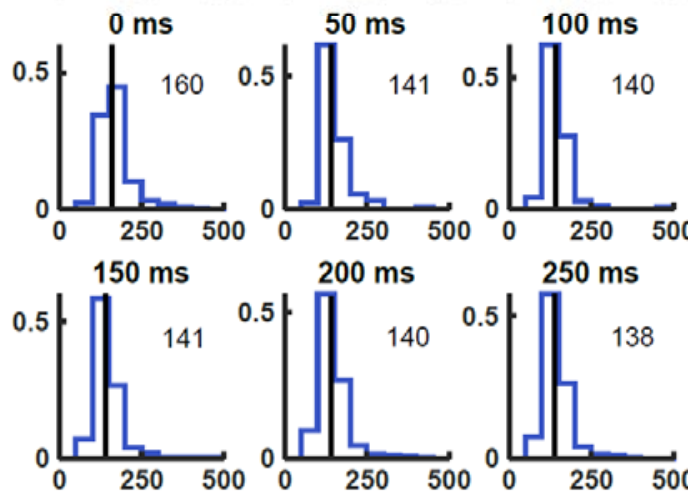

$200 \mathrm{~ms}$
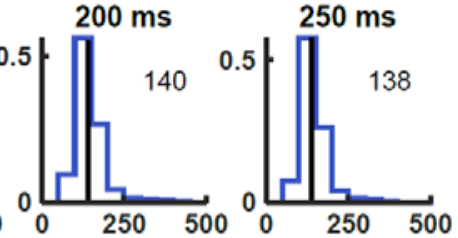

$350 \mathrm{~ms}$

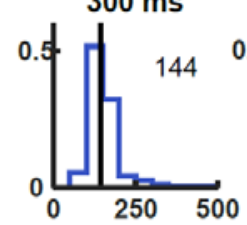

892

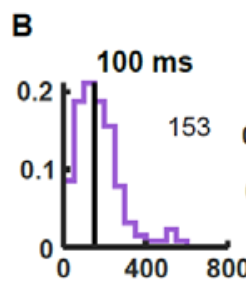

ODR
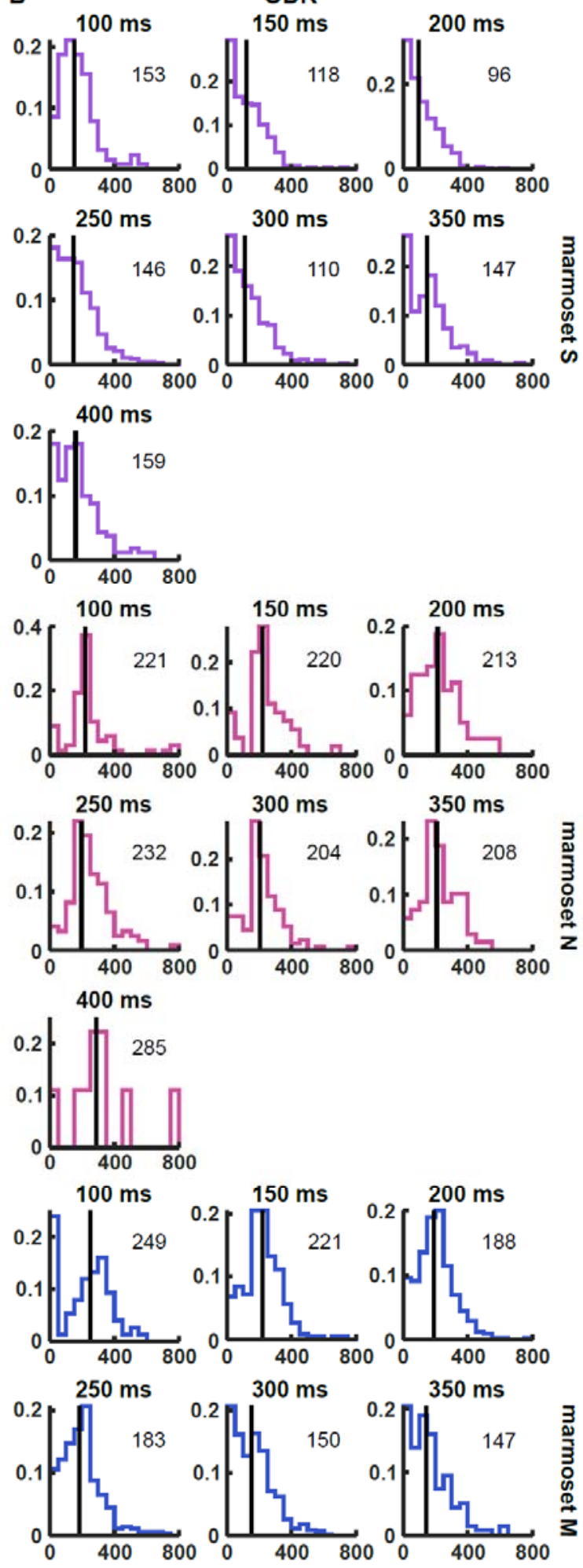

$400 \mathrm{~ms}$

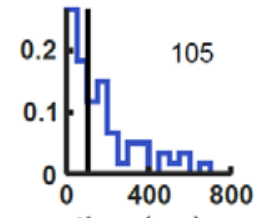

Saccade response time $(\mathrm{ms})$ 
893 Supplementary Figure 2 Saccade response time (SRT) from the successful trials of each gap 894 period for the gap task and each delay period for the ODR task. We plotted the SRT of the 895 answering saccades of the successful trials regardless of the target eccentricity. (A) Gap task. Note 896 that in all three marmosets, the SRT histogram for the $0 \mathrm{~ms}$ gap period had rather a long tail. When 897 the gap period increased to $150 \mathrm{~ms}$, the tail of the histogram got shorter. There was no express

898 saccade observed in all three marmosets. (B) ODR task. Note that in all three marmosets, extra short899 latency saccades $(<70 \mathrm{~ms})$ were observed in all delay periods. The medians of the SRT for each plot 900 were indicated in each plot in black.

901

902 